\title{
Adaptive speciation theory: a conceptual review
}

\author{
Franz J. Weissing • Pim Edelaar • G. Sander van Doorn
}

Received: 8 May 2010 /Revised: 17 November 2010 /Accepted: 22 November 2010 /Published online: 5 January 2011

(C) The Author(s) 2010. This article is published with open access at Springerlink.com

\begin{abstract}
Speciation - the origin of new species-is the source of the diversity of life. A theory of speciation is essential to link poorly understood macro-evolutionary processes, such as the origin of biodiversity and adaptive radiation, to well understood micro-evolutionary processes, such as allele frequency change due to natural or sexual selection. An important question is whether, and to what extent, the process of speciation is 'adaptive', i.e., driven by natural and/or sexual selection. Here, we discuss two main modelling approaches in adaptive speciation theory. Ecological models of speciation focus on the evolution of ecological differentiation through divergent natural selection. These models can explain the stable coexistence of the
\end{abstract}

Communicated by Guest Editor J. McNamara

Franz J. Weissing and G. Sander van Doorn had contributed equally to this work.

This contribution is part of the Special Issue 'Mathematical Models in Ecology and Evolution: Darwin 200' (see Marshall et al. 2010).

\section{F. J. Weissing $(\square)$}

Theoretical Biology Group, Centre for Ecological

and Evolutionary Studies, University of Groningen,

Kerklaan 30,

9751 NN Haren, The Netherlands

e-mail: f.j.weissing@rug.nl

\section{P. Edelaar}

Estacion Biologica de Doñana - CSIC,

c/Americo Vespucio $\mathrm{s} / \mathrm{n}$,

41092 Sevilla, Spain

e-mail: edelaar@ebd.csic.es

\section{G. S. van Doorn}

Department of Behavioural Ecology, Institute of Ecology and Evolution, University of Bern,

Wohlenstrasse 50a,

CH-3032 Hinterkappelen, Switzerland

e-mail: sander.vandoorn@iee.unibe.ch resulting daughter species in the face of interspecific competition, but they are often vague about the evolution of reproductive isolation. Most sexual selection models of speciation focus on the diversification of mating strategies through divergent sexual selection. These models can explain the evolution of prezygotic reproductive isolation, but they are typically vague on questions like ecological coexistence. By means of an integrated model, incorporating both ecological interactions and sexual selection, we demonstrate that disruptive selection on both ecological and mating strategies is necessary, but not sufficient, for speciation to occur. To achieve speciation, mating must at least partly reflect ecological characteristics. The interaction of natural and sexual selection is also pivotal in a model where sexual selection facilitates ecological speciation even in the absence of diverging female preferences. In view of these results, it is counterproductive to consider ecological and sexual selection models as contrasting and incompatible views on speciation, one being dominant over the other. Instead, an integrative perspective is needed to achieve a thorough and coherent understanding of adaptive speciation.

Keywords Speciation models · Prezygotic isolation · Postzygotic isolation - Reinforcement - Disruptive selection - Evolutionary branching $\cdot$ Competitive speciation - Sexual selection - Fisherian runaway process . Good-genes models · Condition-dependent ornament

\section{Introduction}

Recently, the scientific world celebrated the 150th anniversary of the release of Charles Darwin's seminal book On the Origin of Species by Natural Selection. Darwin's ideas 
(Darwin 1859; Darwin 1871) on natural and sexual selection have inspired generations of biologists, and we have now a fairly good comprehension of how selection acts within populations and how populations are transformed under the influence of selection pressures. It is important to realise, however, that much of our understanding of selection-induced changes relates to the process of anagenesis, the gradual evolution of whole populations. A similar understanding of the role of selection in cladogenesis, the splitting of species into reproductively isolated units, is largely lacking. Despite of its title, the Origin did actually not contribute much to resolving the question whether and how speciation is driven by natural selection. A sound understanding of speciation is of key importance for evolutionary theory, since the birth of new species is the crucial link between micro-evolution (that mainly occurs at or below the species level) and macro-evolutionary processes like adaptive radiations that largely occur above the species level. If speciation tends to be adaptive, that is, driven by directional forces like natural or sexual selection, then one could hope for achieving an overarching adaptive theory including both micro- and macro-evolution.

Darwin (Origin, chapter 4) envisaged speciation as the result of two processes: selection for diversification allowing the exploitation of previously unused opportunities, and the extinction of intermediate forms as a consequence of severe competition among these forms. Hence, according to Darwin, selection plays a major role in the speciation process. In fact, his view comes close to modern ideas on competitive speciation to be discussed below. However, Darwin's verbal arguments are often vague and not always convincing, partly because of his pre-Mendelian ideas on inheritance. It is partly for this reason that the founding fathers of the 'Modern Synthesis' largely discarded Darwin's view on speciation (Mayr and Provine 1998), giving nonselective factors like geographic isolation a much more prominent role than selection.

Ever since Darwin, theoretical arguments have played an important role in debates on the causes of speciation. In the second half of the 20th century, more than 100 mathematical models have been developed to study the role of selection in speciation with gene flow (reviewed in Kirkpatrick and Ravigné 2002). Although many of these models demonstrate that selection-driven speciation is possible in principle, they typically lead to the conclusion that selection-driven speciation will only occur under highly specific conditions or for rather extreme parameter combinations (Felsenstein 1981). Accordingly, speciation models seemed to suggest that adaptive speciation is a rare and unlikely phenomenon. This conclusion is challenged by two recent developments in speciation theory, which seem to suggest that natural and sexual selection can be more powerful in the creation of new species than the traditional models seem to suggest. First, a variety of ecological speciation models has been developed (Dieckmann et al. 2004) that show that Darwin's intuitive notion of competitive speciation can be given a theoretical underpinning. These models are based on a dynamic view of natural selection, allowing selection to switch from stabilising to disruptive in the same coherent framework. The realisation that Darwinian fitness is highly context dependent and dynamic, and that in a diversity of settings, natural selection can drive a population toward a regime of ongoing disruptive selection, makes selection-driven speciation more plausible than in traditional models. Second, various sexual selection models of speciation (Ritchie 2007) give disruptive sexual selection a prominent place in the speciation process. Several studies (e.g., Turner and Burrows 1995; Higashi et al. 1999) demonstrate that, under specific circumstances, sexual selection may lead to the divergence of female preferences within a single population, eventually leading to reproductive isolation.

Both developments have initiated a fierce debate in the scientific literature. Models of speciation driven by disruptive selection are inherently complex and therefore have to make many simplifying assumptions. The question therefore arises whether the results of these models are general and representative for real-world systems, or whether instead, they mainly reflect modelling details or the choice of parameters and initial conditions. In case of ecological speciation models, the analysis is often based on concepts of adaptive dynamics theory, which have been heavily criticised (e.g., Waxman and Gavrilets 2005) and defended (e.g., Doebeli and Dieckmann 2005). The representativeness of simulation models of ecological speciation have been questioned (e.g., Gavrilets 2004; but see Doebeli and Dieckmann 2005) because of their assumptions on the genetic architecture of traits, their choice of parameters like mutation rate or population size, and their assumptions underlying the evolution of assortative mating (Matessi et al. 2001). Likewise, models of speciation through disruptive sexual selection have been criticised (e.g., Van Doorn and Weissing 2001; Arnegard and Kondrashov 2004; Van Doorn et al. 2004) because of their highly special initial conditions and the lack of stability of the incipient species in the face of ecological competition. For non-specialists (and not only for them!), it is increasingly difficult to judge the scope and validity of the arguments and counterarguments in this debate. This is partly a consequence of the high degree of technical sophistication required for setting up and analysing adaptive speciation models. Moreover, proponents and opponents tend to base their views on model variants that differ to such an extent in their assumptions that the model outcomes are not directly comparable. 
In situations like these, it is often impossible to say that one model variant is "inherently" better than an alternative one. Selection-driven speciation results from the intricate interplay of processes at the phenotypic level (where natural and sexual selection are operating) and at the genetic level (where, for example, linkage disequilibria have to evolve between ecological and mating characters), and models focusing simultaneously on both levels tend to be intractable. The more traditional speciation models tend to have their focus on genetic processes and are often quite sophisticated in this respect. On the downside, they often make simplistic assumptions on selection and mating, which cannot easily be given a mechanistic interpretation and which typically lack a population dynamical underpinning. In contrast, the adaptive dynamics school tends to derive fitness from first principles; fitness "emerges" from mechanistic and population dynamical considerations (Metz 2008). On the downside, the assumptions on the underlying genetics are often simplistic and unrealistic. It is important to realise that both approaches have their virtues and their shortcomings; and that each approach sheds light on different aspects of the process of speciation. Although the diversity of models and model outcomes may look confusing, a pluralistic approach may actually be the best research strategy for achieving robust insights. Speciation theory is the prototype example of a research field where scientific truth can only be approached through the "intersection of independent lies" (Levins 1966).

This contribution is an attempt to present some basic concepts of adaptive speciation theory in an intuitive, nontechnical way, thereby helping the uninitiated to find their way through the thicket of arguments currently used in this field. We do not intend to give a comprehensive review of the field of speciation theory, since recent reviews are readily available (e.g., Turelli et al. 2001; Kirkpatrick and Ravigné 2002; Gavrilets 2003, 2004; Coyne and Orr 2004; Dieckmann et al. 2004; Bolnick and Fitzpatrick 2007; Ritchie 2007). Instead, this is an informal discussion of a few models that highlight current thinking in speciation theory. Being the elaboration of a conference talk, this contribution is biased to our own work, and it does not address important aspects, such as the explanatory power of speciation models when applied to well-studied real-world systems (cichlids: Lande et al. 2001; Gavrilets et al. 2007; sticklebacks: Berner et al. 2008; guppies: Labonne and Hendry 2010; walking sticks: Nosil and Yukilevich 2008; snails: Sadedin et al. 2009; sea urchins and abalone: Van Doorn et al. 2001; palms: Gavrilets and Vose 2007). Throughout, we will focus on adaptive routes to speciation, that is, on speciation scenarios where the evolution of reproductive isolation and/or ecological differentiation is directly driven by selection (Baker 2005).
This paper is structured as follows. In the next section, we will start with a classification of speciation models. Here, we will clarify our usage of the term 'adaptive speciation,' and the relationship between 'traditional' (i.e., allopatric), 'ecological', and 'sexual selection' models of speciation. We will then turn to ecological models of speciation, where disruptive natural selection leads to ecological differentiation and the evolution of assortative mating. Subsequently, we will review some sexual selection models of speciation, where Fisherian runaway selection leads to diverging preferences and subsequently to prezygotic isolation. After having assessed the merits and shortcomings of both types of model, we consider a combined model including both disruptive natural selection and diversifying sexual selection. Perhaps surprisingly, it will turn out that both factors, when acting on their own, are not able to induce speciation. Only when mate choice has an ecological component, speciation is readily achieved. Putting mate choice into an ecological setting is also the theme of the last section, where we consider the evolution of female preferences for male ornaments signalling the degree of adaptation to the local ecological conditions. In such a scenario, sexual selection and disruptive natural selection mutually reinforce each other and relatively easily bring about the simultaneous evolution of ecological differentiation and prezygotic isolation. We conclude this paper with a plea for an integrative approach to the speciation problem. The tendency to consider potential speciation mechanisms in isolation is perhaps understandable, but neither the assumptions nor the results of 'single factor' speciation models should be considered representative for the origin of species in the real world.

\section{A classification of speciation models}

If species are defined according to the biological species concept as "groups of actually or potentially interbreeding natural populations, which are reproductively isolated from other such groups" (Mayr 1942; see also De Queiroz 2005), the evolution of reproductive isolation is the crucial step in the speciation process. To this end, we can distinguish between of pre- and postzygotic isolation mechanisms. Many evolutionary biologists (but see Presgraves 2010) consider a speciation process only as 'completed' once the incipient species have become prezygotically isolated from each other, that is, once hybridisation is prevented because of strong assortative mating. Accordingly, most speciation models try to explain the evolution of assortative mating preventing the interbreeding between nascent species. From an ecological point of view, reproductive isolation is, however, not sufficient for achieving a speciation event of lasting effect. In addition, ecological differentiation of the 
incipient species is required in order to allow their coexistence in a longer-term perspective.

Figure 1 illustrates how reproductive isolation and ecological coexistence are achieved in three major classes of speciation models. The orange boxes and arrows indicate the classical view of speciation, as championed by Ernst Mayr (1942, 1963) and other architects of the Modern Synthesis. According to this view, speciation is typically preceded by externally induced reproductive isolation (e.g., by the advent of geographical barriers preventing gene flow between various parts of a population). Hence, at least initially assortative mating and prezygotic isolation come for free. However, the separated populations will only be considered 'true' species if they do not interbreed when the external barrier is removed again. There are various theories how this can be achieved. Virtually, all argue that the independent evolution of two separated parts of a population will lead to population divergence, which eventually will result in low-fitness hybrids, that is, postzygotic isolation. Once hybrids have low fitness, there should be selection against hybridisation, that is, selection for prezygotic isolation. Accordingly, a primary phase of (externally caused) reproductive isolation is followed by secondary postzygotic isolation, which, via a process called 'reinforcement' (Servedio 2004; Servedio and Noor 2003), results in secondary (internally caused) prezygotic isolation that keeps the incipient species apart even if they come into secondary contact again.

Selection does not necessarily play a major role in the evolution of (secondary) reproductive isolation. It can be

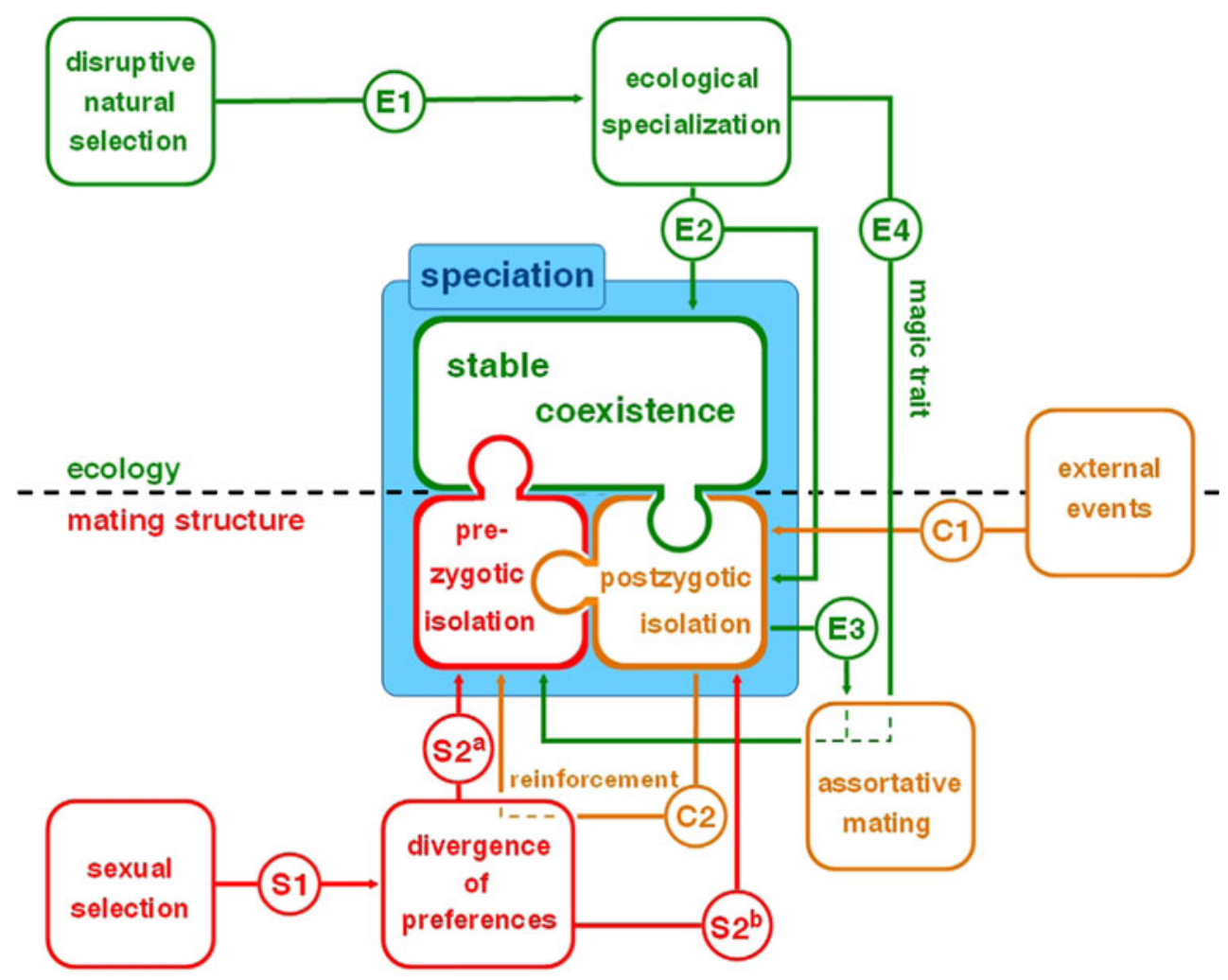

Fig. 1 Schematic classification of speciation models. Each speciation model has to explain the evolution of (post- and prezygotic) reproductive isolation and the evolution of ecological differentiation allowing the stable coexistence of the incipient species. (C) In classical models of speciation (orange boxes and arrows), reproductive isolation is initially caused by external events. The two isolated populations evolve separately, thereby gradually diverging from each other. Divergence is associated with the accumulation of genetic or other incompatibilities which result in a fitness reduction in hybrids and, hence, postzygotic isolation (C1). As a consequence, mechanisms preventing hybridisation are selectively favoured once the external cause of reproductive isolation is removed (reinforcement, C2). Accordingly, externally induced prezygotic isolation is replaced by internal prezygotic isolation mechanisms. Only few classical models address the coexistence problem. (E) In ecological models of speciation (green boxes and arrows) disruptive natural selection leads to ecological differentiation (E1), that is, to the evolution of distinct ecotypes that stably coexist in the face of ecological competition (E2). Reproductive isolation can evolve via two routes. Differentiation can be associated with postzygotic isolation, since hybrids may have reduced fitness because of their disfavoured intermediate phenotype. In this case, prezygotic isolation can subsequently evolve via reinforcement (E3). Alternatively, the traits leading to ecological differentiation are 'magic traits' in the sense that they directly lead to assortative mating and, hence, prezygotic reproductive isolation (E4). (S) In sexual selection models of speciation (red boxes and arrows) Fisherian runaway selection leads to the divergence of mating preferences (S1) which induce both prezygotic isolation ( $\mathrm{S}^{a}$; because females differing in preferences will mate with different types of males) and postzygotic isolation $\left(\mathrm{S}^{b}\right.$; because hybrids do not match the preferences of either type of female). At present, only few sexual selection models address the coexistence problem 
argued (Turelli et al. 2001) that the evolution of secondary isolation mechanisms is an inevitable consequence of primary isolation. As long as two populations are isolated, there is no selection enforcing reproductive compatibility between the populations. Hence, any such compatibility mechanisms will eventually decay, leading to sister species that are reproductively incompatible. Two specific models for the accumulation of reproductive incompatibilities are the Dobzhansky-Muller model concerning the negative effects of gene substitutions in novel genetic backgrounds (Orr 1995; Orr and Turelli 2001) and Gavrilets' model (1999, 2004) concerning evolution on 'holey' fitness landscapes.

However, the evolution of secondary reproductive isolation in diverging populations is speeded up considerably if it is driven by diversifying selection. For example, divergent natural selection in two populations facing slightly different ecological conditions leads to a rapid reduction of hybrid fitness, because of the accumulation ecological incompatibilities (Coyne and Orr 2004) and the more efficient accumulation of genetic incompatibilities (Unckless and Orr 2009). As noticed by Lande (1981), sexual selection can also speed up the evolution of both post- and prezygotic isolation. In fact, the direction of sexual selection can be quite arbitrary if it is driven by a Fisherian runaway process (Andersson 1994). Accordingly, it is easy to conceive that mating preferences diverge in two isolated populations. When the primary cause of isolation is removed, the incipient species may have become secondarily isolated, both because of prezygotic (since members of one population are not found attractive by members of the other population) and postzygotic isolation (since hybrid offspring have low mating success in both populations). Intraspecific arms races caused by genetic or sexual conflict are an even more potent selective force driving isolated populations apart (Parker and Partridge 1998; Gavrilets 2000).

Hence, even in the classical models of speciation, natural and sexual selection can play an important role, be it in the evolution of postzygotic isolation (low hybrid fitness) or in the subsequent evolution of prezygotic isolation through reinforcement (Dobzhansky 1940; Kirkpatrick and Servedio 1999; Servedio and Noor 2003). However, speciation is not initiated by selection, and selection only comes into play after parts of a population have become isolated due to external events. For this reason, we do not consider speciation via the classical model 'adaptive' and we reserve the term adaptive speciation to those events where directional forces like natural or sexual selection are really crucial for the initiation and completion of speciation (Baker 2005). On the other hand, our usage of 'adaptive' speciation is much broader than that of (Dieckmann et al. 2004), who reserve the term for speciation driven by highly specific forms of natural selection (see Gavrilets 2005).
Figure 1 also illustrates the two main adaptive routes to speciation, where either ecological differentiation or reproductive isolation is directly driven by disruptive selection. In ecological models of speciation (green boxes and arrows in Fig. 1), disruptive natural selection leads to differentiation in ecotypes, which in principle can stably coexist due to this differentiation. As we will discuss in the next section, the main problem of ecological models is to explain the evolution of assortative mating and, as a consequence, reproductive isolation. In most sexual selection models of speciation (red boxes and arrows), Fisherian runaway selection leads to diverging mating preferences and subsequently to prezygotic isolation. As we will see below, the main problem of these models is to explain the stable coexistence of the newly evolved species in the face of ecological competition.

\section{Speciation through natural selection}

The idea underlying ecological speciation is simple and straightforward. Imagine a situation with two or more ecological niches, corresponding to fitness peaks on an adaptive landscape, where ecological specialists focussing on one of the niches have on average a higher fitness than a generalist. In such a situation, there is selection for ecological specialisation. Let us further make the plausible assumption that matings between two different specialists result in offspring with lower fitness, since these offspring are not adapted to any of the niches. Then there is selection for assortative mating to prevent these unproductive matings. If assortative mating is strong enough, an original species of generalists can evolve into two reproductively isolated species of specialists.

Starting with Maynard Smith (1966), there have been numerous attempts to model the scenario sketched above (reviewed by Kawecki 2004). It soon turned out there are several important obstacles on the road to speciation (see Van Doorn 2004, chapter 1, for a detailed account). As noticed already by Mayr (1963), sustained disruptive selection, operating over many generations, is only achieved under highly restrictive and unrealistic conditions in classical population genetical models. Yet, even a stable regime of disruptive selection will typically not result in polymorphism but lead to the evolution of a single ecological specialist instead. Finally, if polymorphism is maintained, the evolution of assortative mating with respect to the ecological character under disruptive selection may be difficult to achieve. If the ecological character and the traits controlling assortative mating are induced by different sets of genes, the required association between mating traits and ecological traits may not easily evolve, since it is counteracted by recombination (Udovic 1980; Felsenstein 
1981). Many evolutionary biologists have therefore concluded that speciation purely driven by disruptive selection (i.e., in the absence of external isolation mechanisms) is unlikely, unless the ecological character under disruptive selection happens to be a 'magic trait' (Gavrilets 2004) that also controls mating.

This conclusion may have been premature. The traditional models on the evolutionary implications of disruptive natural selection were based on rather simple ecological (and genetic) assumptions. They typically involved two discrete ecological niches, a single ecological trait encoded by one gene locus with two alleles and relatively simple fitness relationships. Already in 1978, Rosenzweig argued by means of a graphical approach that the first two of the above-mentioned obstacles (the establishment of a stable regime of disruptive selection, and the maintenance of polymorphism) can be overcome if one adopts a more dynamic view of a fitness landscape. Rosenzweig's ideas precede the developments in modern selection theory, which explicitly take account of the change of a fitness landscape during the course of evolution. We will here discuss the concept of evolutionary branching developed in adaptive dynamics theory (e.g., Geritz et al. 1998, 2004), but very similar concepts were developed in other areas of selection theory, including population and quantitative genetics (Abrams et al. 1993a, b; Day 2005).

Figure 2 illustrates the prototype example for evolutionary branching that forms the basis of the model of competitive speciation by Dieckmann and Doebeli (1999). Instead of starting with a discrete number of ecological niches, this model considers a consumer species that forages on a continuous spectrum of resources, in Fig. 2, illustrated by seeds varying in size. In the model, the resource distribution is externally given (green solid line in Fig. 2). The consumers differ in an 'ecological character,' which is Fig. 2 is illustrated by bill size. The fitness of each type of consumer is determined by its food intake, which depends on two factors. First, the consumers differ in their ability to utilise various parts of the resource distribution in relation to their ecological character. For example, a largebilled bird is efficient in utilising seeds of large size (illustrated by the dashed line in Fig. 2a), but not efficient in exploiting seeds of small size. Second, the food intake rate depends on the amount of competition experienced from other consumers in the population. All other things being equal, a consumer of a given ecotype will have a lower food intake rate when many conspecifics compete for the same resources (i.e., are of a similar ecotype) than when the other members of the population focus on a different part of the resource spectrum. This latter factor makes fitness frequency dependent, that is, the fitness of a consumer depends on the distribution of ecological characters in the population. Accordingly, the fitness landscape is
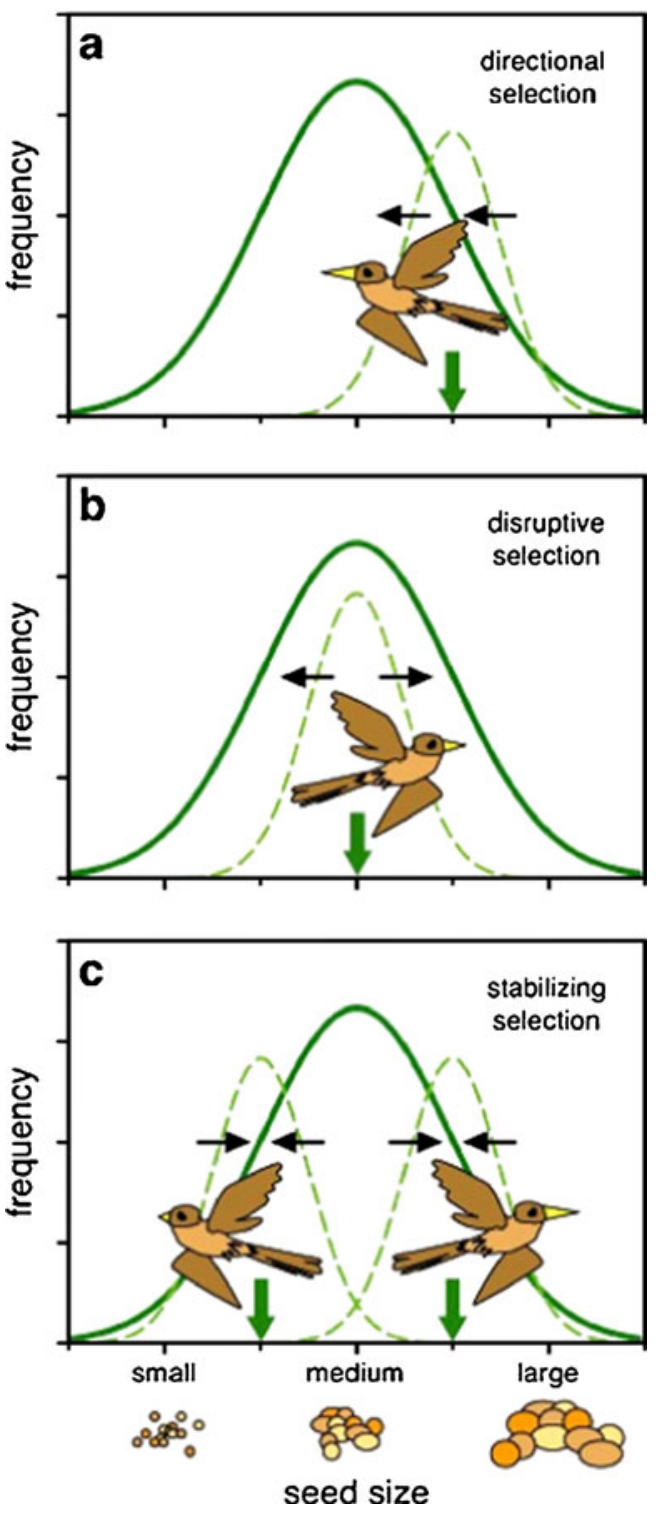

Fig. 2 Evolutionary branching in ecological speciation models. Individuals compete for resources, which, in this example, are represented by seeds of different sizes. Depending on its phenotype (bill size), an individual can utilise only a limited fraction (indicated by the dashed resource utilisation curve) of the full distribution of resources (solid curve). a When the mean bill size in the population does not match the most abundant resource, directional selection (arrows) pushes the phenotype to an intermediate value. This eventually leads to strong competition for the most abundant seeds (b). Since resources in the tails of the distribution are left unexploited, selection favours individuals with either small or large beak sizes. c Under the influence of disruptive selection, the population splits into two ecological specialists that evolve an optimal compromise (arrows indicate that selection eventually becomes stabilising) between foraging on the most abundant seeds and avoiding competition

dynamic, since any change in the distribution of ecological characters will lead to a change in fitness of all ecotypes.

The dynamic nature of the fitness landscape has important implications. Assume that, as illustrated in Fig. 2a, the consumer population consists of individuals 
with an ecotype (here: large-billed birds) that does not match the part of the resource spectrum where resources are most abundant (here: seeds of medium size). Then ecotypes with a better match (here: medium-billed birds) will have a selective advantage and eventually replace the original ecotype. Hence, selection is directional, and it will proceed until the situation in Fig. $2 b$ is reached, where the dominant ecotype in the population perfectly matches the dominant type of resource. Now several things can happen. If the dominant ecotype in the situation of Fig. $2 b$ has a broad 'resource utilisation curve' (symbolised by the dashed line), it can cover the whole resource spectrum. In a case like this, no alternative ecotype can invade, and there is stabilising selection for the ecotype matching the peak of the resource distribution. Figure $2 b$ illustrates the opposite case where the resource utilisation curve is narrow when compared to the resource distribution. Now the resident population is very efficient in exploiting the dominant resource (mediumsized seeds), but it leaves major parts of the resource spectrum unexploited. Ecotypes specialised on the unexploited part of the resource spectrum (i.e., small- and largebilled birds) actually have a selective advantage when rare, since they are less affected by competition by conspecifics. Yet, they cannot take over the population, since in that case directional selection would shift the consumer population back to the original state in Fig. 2b. Instead, selection favours 'evolutionary branching,' that is, the replacement of the originally monomorphic population by a polymorphism of two (or more) ecotypes that together exploit a broader range of the resource spectrum.

In an asexual population, evolutionary branching is indeed sufficient to achieve the transition from Fig. $2 b$ to $\mathrm{c}$, that is, to induce the evolution of several coexisting ecotypes. In sexual populations, the situation is more complicated, since random mating between the branches will lead to hybrids of intermediate ecotype, thereby counteracting the tendency towards ecological specialisation. In addition to evolutionary branching, assortative mating has to evolve, which prevents hybridisation between differently specialised ecotypes. As shown by Dieckmann and Doebeli (1999), assortative mating can indeed get off the ground in various ways, leading to the stable coexistence of reproductively isolated ecotypes and, hence, speciation. From this, Dieckmann and Doebeli conclude that the verbal arguments of Darwin (1859) and the graphical approach of Rosenzweig (1978) were essentially correct and that competitive speciation completely driven by disruptive natural selection is indeed a plausible scenario.

For competitive speciation to occur, it is crucial that the fitness landscape is not fixed, but that selection is frequency dependent. A population is only subject to disruptive selection if it happens to be located in a "fitness valley."
If the fitness landscape is fixed, fitness valleys also have a fixed location. It is quite unlikely in the first place that a population finds itself in such a valley. Even if it does, the valley and, hence, the regime of disruptive selection will rapidly be left, since selection tends to increase fitness ("climbing uphill the fitness landscape"). In contrast, frequency-dependent selection corresponds to a climb on a "wiggling landscape" where new fitness peaks and valleys appear in the course of evolution (McNamara and Weissing 2010). If the requirements for evolutionary branching are met (as in the Dieckmann-Doebeli model), a fitness valley appears in a natural way on the evolutionary trajectory of a population. In other words, selection shifts the population to an emerging fitness valley and keeps it in the corresponding regime of disruptive selection for extended periods of time. The latter is important, since it provides time and opportunity for assortative mating to evolve.

Although the early literature on speciation (e.g., Udovic 1980) already realised the importance of frequencydependent selection, we consider evolutionary branching an important conceptual breakthrough. Compared to the more traditional speciation models, the adaptive dynamics approach has two main advantages. First, the fitness of the various genotypes is not a priori given by some fixed parameters or functional relationships, but instead derived from population dynamical considerations. As a consequence, adaptive dynamics models are to a much higher degree "ecologically consistent" than earlier population genetical approaches. Moreover, the derivation of fitness from more basic underlying principles regularly reveals new sources of frequency dependence that in classical models remained undetected. Second, adaptive dynamics theory provides simple and transparent criteria allowing to judge in a relatively straightforward way whether, and under what conditions, a given ecological scenario can lead to evolutionary branching. The above example of branching induced by the competition of a consumer species for resources may appear specific, but in the last decade a multitude of ecological scenarios have been identified where evolutionary branching is expected to occur (Dieckmann et al. 2004; Doebeli et al. 2005). Not surprisingly, these scenarios can as easily give rise to ecological speciation (Doebeli and Dieckmann 2000, 2005) as the example in Fig. 2. Accordingly, the adaptive dynamics approach of Dieckman and Doebeli shifts the attention away from modelling details and towards robust features of the ecological interaction structure.

Despite these advantages, the Dieckmann-Doebeli approach has attracted much criticism and ignited a lively debate in the scientific literature. One point of concern is related to the fact that speciation is not the only possible outcome when a population is subject to evolutionary 
branching (Rueffler et al. 2006). Whether or not speciation will occur does to a large extent depend on the model structure and to the constraints imposed on the availability of genetic variation. The situation of Fig. $2 b$, for example, does only lead to disruptive selection because the resource utilisation curve of the consumers is narrow when compared to the resource spectrum. In the Dieckmann-Doebeli model, the width of the resource utilisation curve is fixed and not able to evolve. If this were not the case, it is conceivable that selection would lead to the evolution of a single ecological generalist (with a broad resource utilisation curve) rather than several coexisting specialists. The evolution of sexual dimorphism (where males and females specialise on different parts of the resource spectrum) or of phenotypic plasticity would also be an alternative to speciation (Bolnick and Doebeli 2003; Van Dooren et al. 2004)

The main critique on the Dieckmann-Doebeli model is directed at the claim that reproductive isolation will evolve relatively easily when the conditions for evolutionary branching are satisfied. Several studies suggest that the conditions for the evolution of strong assortative mating are actually quite restrictive (e.g., Matessi et al. 2001; Gourbiere 2004; Gavrilets 2004, 2005; Waxman and Gavrilets 2005; Polechová and Barton 2005; but see also Doebeli and Dieckmann 2005; Doebeli et al. 2005). Part of the debate centres on technical aspects, for example the question whether speciation will still occur for more realistic initial conditions, an increased number of loci, more realistic mutations rates, the inclusion of mutations of larger effect size, or the removal of constraints on the range of possible phenotypes (see Gavrilets 2004; Polechová and Barton 2005; Bürger et al. 2006). A more fundamental line of attack concerns the implementation of assortative mating. In the Dieckmann-Doebeli model, no costs are associated with assortative mating, while it seems plausible that choosiness always involves at least some costs (Andersson 1994). In the initial phase of speciation, selection in favour of assortative mating is rather weak, and even small costs might prevent that assortative mating gets off the ground. In addition, the fitness penalty associated with assortative mating should be frequency dependent, since rare phenotypes will have more difficulty in finding a mate of similar phenotype than common ones. Hence, costly mating is associated with stabilising selection, which at least to some extent should counteract the disruptive selection driving the speciation process. It is therefore not too surprising that studies including costs of assortative mating (e.g., Matessi et al. 2001; Bolnick 2004; Kirkpatrick and Nuismer 2004; Schneider and Bürger 2006; Pennings et al. 2008; Kopp and Hermisson 2008; Ripa 2009) arrive at the conclusion that even small costs frustrate the evolution of reproductive isolation, or that in the presence of costs, speciation is not completed since assortative mating only evolves to a moderate level.

We can conclude that the Dieckmann-Doebeli model has certainly not removed all obstacles on the road to selectiondriven speciation. Several studies (e.g., Polechová and Barton 2005; Ripa 2009) even come to the conclusion that competitive speciation is more difficult (or even impossible) to achieve in scenarios with a continuous unimodal resource distribution. Although the debate is far from over, consensus emerges that ecological speciation driven by evolutionary branching is less likely than the original Dieckmann-Doebeli model seems to suggest.

\section{Speciation through sexual selection}

As illustrated by the above discussion, the evolution of reproductive isolation is still a weak aspect of ecological speciation models. In the realm of ecological models, most attempts to study reproductive isolation are based on submodels for the evolution of assortative mating ('like mates with like'). The simplest models (and the ones most easily leading to speciation) are those where assortative mating is directly based on the ecological character under disruptive selection. This is, for example, a plausible scenario when the ecological trait is related to habitat choice, and mating takes place in the habitat. In general, however, mating will be based on signals that are only loosely related to the ecological characters in question. To address situations like this, more sophisticated models of assortative mating consider the evolution of a marker trait on which assortative mating can be based. As noticed by Felsenstein (1981), speciation is much more difficult to achieve in the latter type of models, since (1) the marker trait needs to remain polymorphic, and (2) the marker trait needs to become genetic correlated with the ecological character under disruptive selection. A variety of assortative-mating models has been comprehensively reviewed by Gavrilets (2004, chapter 10). Most of these models were constructed from the viewpoint of mathematical convenience. From a behavioural point of view, these models (e.g., the ones in Dieckmann and Doebeli 1999) are not very convincing, since they cannot easily be translated into a plausible mechanism of mate choice. The lack of mechanistic detail is perhaps responsible for the fact that most assortative mating models used in speciation theory make the unrealistic assumption that assortative mating has no cost (Schneider and Bürger 2006). As discussed above, the inclusion of such costs has major implications for the evolution of reproductive isolation.

Sexual selection theory provides another approach to the selection-driven evolution of reproductive isolation. Technically speaking, any form of (assortative) mating where 
not all individuals have the same mating success may be subsumed under the heading 'sexual selection' (as many authors do, e.g., Gourbiere 2004; Pennings et al. 2008). Here, we use the term in a more narrow sense, in order to indicate the evolution of female preferences for male ornaments, which may or may not be indicators of genetic or phenotypic quality. In other words, we will focus on some of the standard models of sexual selection theory (Andersson 1994), which are well elaborated and studied, and which tend to be much more 'mechanistic' than the before-mentioned models of assortative mating. Most sexual selection models are more complex than assortative mating models, but they have the considerable advantage that costly female preferences can evolve from scratch, whereas costly assortative mating cannot easily get off the ground.

Fisher $(1930,1958)$ was probably the first to recognise the potential role of sexual selection for the process of speciation. In his seminal book, he describes a "runaway process" where mating preferences for quite arbitrary secondary sexual characters (henceforth called "ornaments") can rapidly evolve in a self-reinforcing way. Fisher realised that runaway sexual selection may be a potential mechanism for rapid speciation, since if a runaway process occurs in an isolated population, the newly evolved preferences and ornaments may immediately lead to prezygotic reproductive isolation. Lande $(1981,1982)$ later formalised these ideas in a quantitative genetic model, again stressing the importance of Fisherian runaway for speciation (see also Lande and Kirkpatrick 1988).

The ideas of Fisher and Lande were mainly applied in the context of allopatric or parapatric speciation, that is, in the presence of externally induced reproductive isolation. More recently, several simulation studies (Wu 1985; Turner and Burrows 1995; Van Doorn et al. 1998; Higashi et al. 1999) led the authors to conclude that sexual selection on its own (i.e., in sympatry) is able to induce speciation. Under specific circumstances, female preferences and the corresponding male ornaments can diverge, thereby leading to reproductive isolation and speciation. Based on a specific model for speciation induced by the evolution of gameterecognition proteins, Van Doorn et al. (2001) argue that the mechanism underlying the divergence of male and female mating types is very similar to the divergence of ecotypes due to evolutionary branching. Their argument is quite general and also applies to speciation via the divergence of female preferences and male ornaments (Van Doorn and Weissing 2001).

This is illustrated in Fig. 3. Assume for the moment that there is a fixed spectrum of female preferences, illustrated by the solid bell-shaped curves in Fig. 3. The dashed lines corresponding to specific variants of the male ornament indicate the propensity of females with a given preference
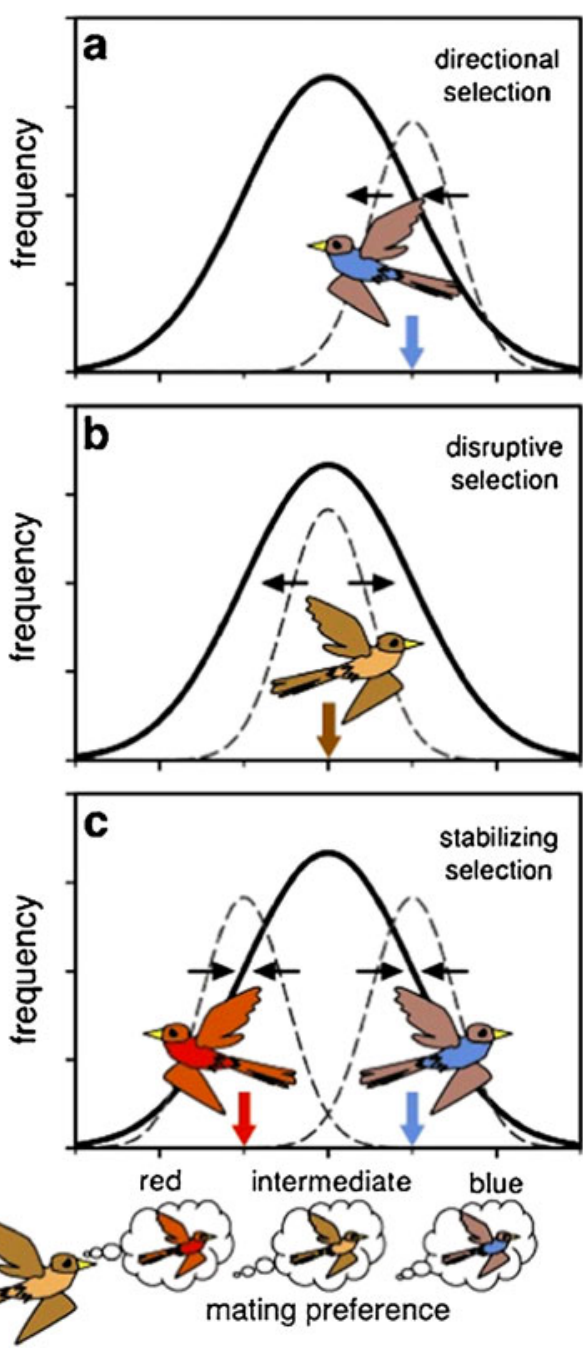

Fig. 3 Evolutionary branching of male ornaments in case of a broad distribution of female preferences. Competition for access to mates among males is comparable (and mathematically equivalent) to ecological resource competition (Fig. 2). Males with a given phenotype (here plumage coloration) may only be acceptable as mates to a subset of the females in the population (indicated by the grey dashed curves; analogous to the resource utilisation curve in Fig. 2), if the population features a broad distribution of females preferences (solid black curve; analogous the resource distribution in Fig. 2). a Under the influence of directional selection the male ornament evolves to match the most common female preference. b After directional selection has vanished, the ornament is subject to disruptive selection if the distribution of preferences is broad. c Indirect competition for access to mates between the males may then induce the population to split into different male types (here, red and blue males)

to mate with this type of male. If the males in a population are endowed with an ornament that does not match the modal preference in the female population (Fig. 3a), there will be directional selection on the ornament, shifting the ornament toward the peak of the female preference distribution. Once this peak is reached (Fig. 3b), the course of further evolution will depend on the variation of preferences in the female population. If female preferences 
show a narrow distribution, the males present in the population can mate with all types of female. As a consequence, there will be stabilising selection on the male ornament. If, however, the distribution of female preferences is broader than the 'utilisation curve' associated with the ornament (the situation depicted in Fig. 3b), the extreme preferences remain 'unexploited' by the males. As in the context of resource competition (Fig. 2b), the combination of intense competition among the predominant types (here: competition of males with the predominant type of ornament) and the presence of unexploited opportunities (here: extreme female preferences) leads to disruptive selection on the male ornament. This will eventually lead to the divergence of male ornaments through a process analogous to evolutionary branching (Fig. 3c).

The main difference between Figs. 2 and 3 lies in the fact that female preferences are not static, but coevolving with the male ornaments. If female fitness associated with a certain preference is positively related to the abundance of preferred males, male ornament branching will be followed by female preference branching, that is, the distribution of female preferences will become bimodal, the modes matching the male ornaments (like in the two lower panels in Fig. 4). Since the genes for a given preference become genetically correlated with the genes for the corresponding ornament, the evolved situation corresponds to assortative mating. If assortative mating is strong enough, two reproductively isolated populations result, each with a particular type of female preference and the corresponding male ornament. In line with these verbal arguments, speciation via two diverging runaway processes do indeed readily occur in both simulation and analytical models (e.g., Higashi et al. 1999; Takimoto et al. 2000; Van Doorn and Weissing 2001).

However, as stressed above the presence of sufficient initial genetic variation of female preferences is a prerequisite for the above route to speciation via the divergence of female preferences and male ornaments. How, then, is this variation achieved in the first place? As noticed by Higashi et al. (1999) and shown more generally by Van Doorn et al. (2004), a Fisherian runaway process will typically not result in sufficient variation in preferences. Accordingly, additional factors are required to achieve speciation. Three scenarios for the generation of sufficient variation in preferences have been discussed in the literature.

First, Higashi et al. (1999) considered the possibility that a sudden change in environmental conditions changes the parameters of mate choice in such a way that previously
Fig. 4 Speciation through the joint action of disruptive natural and sexual selection. The three panels show the distribution of an ecological character (bill size; green), a female preference (red) and a male ornament (plumage colour; blue) through 4,000 generations of evolution in an individual based simulation (adapted from Van Doorn and Weissing 2001). This simulation assumes that the distribution of seeds is broad relative to the range of seeds that can be consumed by an individual with a given bill size, such that the population experiences disruptive selection on bill size (as in Fig. 2). In addition, females have specific preferences, favouring diversification of the male ornament (as in Fig. 3). Early on in the simulation, the population becomes variable for all three characters, but speciation occurs only after bill size and mating characters have become statistically associated (after generation 3,000 ). The result is two reproductively isolated species that can coexist since they partially avoid competition for seeds with one another
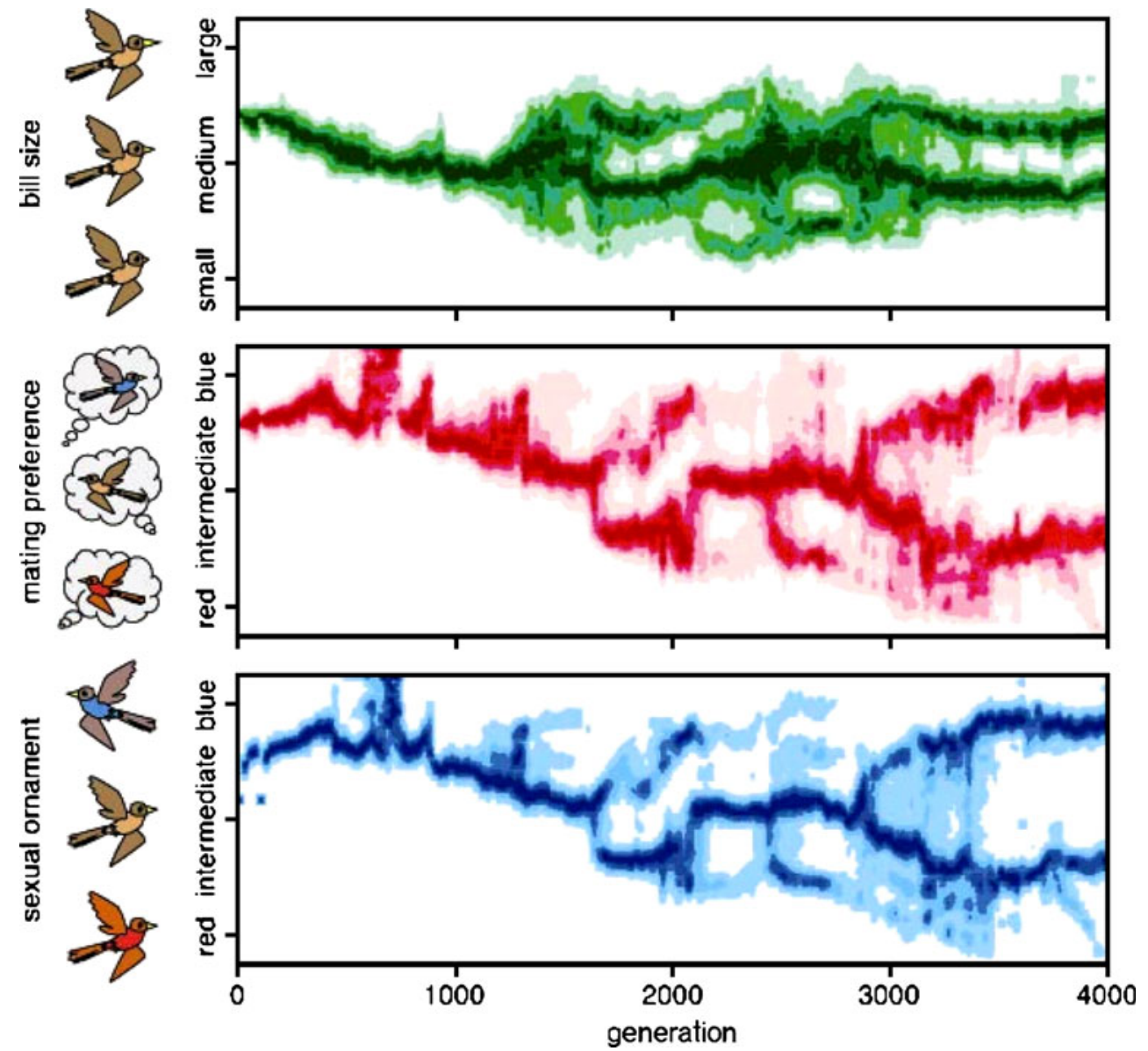
hidden genetic variation of female preferences is suddenly exposed. For example, it has been argued that the decline in haplochromine cichlid diversity in Lake Victoria was caused by the deterioration of underwater light conditions, because the increased turbidity of the water compromised female mate choice based on male coloration (Seehausen et al. 1997). Under turbid conditions, there can be substantial genetic variation in female preferences that never gets expressed, since the females are not able to tell different male colors apart. If the water would suddenly become clear again, this variation would suddenly become expressed, possibly leading to new speciation events as described above. Although this path to speciation through sexual selection is possible in principle, we do not consider it very likely because it will only occur if the change in environment is sudden and large (see Higashi et al. 1999; Fig. 2, Arnegard and Kondrashov 2004).

Second, substantial genetic variation of female preferences can be maintained if selection on preferences is very weak or absent and if new preferences genes are created by mutation at a high rate (e.g., Wu 1985; Takimoto 2002). As argued by Van Doorn et al. (2001), this combination of weak selection and high mutation rates might be responsible for the high speciation rates in marine invertebrates. In general, however, we do not consider this a likely scenario. Moreover, one might argue that speciation in this case is not really adaptive, since it relies on non-adaptive processes like the accumulation of mutations.

Third, a broad distribution of female preferences might be created and maintained by disruptive selection (Van Doorn et al. 2004; see also Lande et al. 2001; Almeida and de Abreu 2003 for models involving mutual mate choice). This typically requires a rather intricate interplay of different selective pressures, at least, if the divergence of mating preferences has to be accompanied by a simultaneous divergence of male ornaments to generate reproductive isolation. The reason is that the conditions for female preference branching are mutually exclusive with the conditions for male ornament branching in standard models of sexual selection (Van Doorn et al. 2004), such that an additional, independent source of disruptive selection on the mating traits is necessary to initiate speciation. Additional disruptive selection can arise from various sources. Van Doorn et al. (2004) present a concrete example where mate choice is combined with disruptive intrasexual selection generated by male-male competition, demonstrating that diversifying selection on female preferences can lead to truly adaptive speciation (that is, speciation fully driven by sexual selection).

We may conclude that speciation through sexual selection, driven by diverging Fisherian runaway processes, is a possibility - at least in theory. However, speciation is either not really adaptive (since it relies on abrupt changes of the environment or on mutation accumulation) or it requires the simultaneous action of multiple sources of selection (diversifying selection on female preferences combined with diversifying selection on male ornaments). Accordingly, the conditions for speciation through sexual selection are much more restrictive than the first models seemed to suggest (see also Arnegard and Kondrashov 2004).

Moreover, we have not yet addressed the coexistence problem (Fig. 1). Even if speciation through diverging female preferences does occur, the disruptive forces involved are typically weak and unable to support the stable coexistence of the incipient daughter species in the face of ecological competition. In fact, the outcome of speciation through Fisherian runaway selection is typically rather labile (e.g., Takimoto et al. 2000). As illustrated by the two lower panels in Fig. 5, in the absence of ecological divergence, the splitting into daughter species is usually only a transient phenomenon. Due to stochastic factors, one of the daughter species may achieve a higher abundance than the other, and the low-abundance species will typically go extinct (Johansson and Ripa 2006).

\section{Interplay of natural and sexual selection}

As argued above, ecological and sexual selection models of speciation both have their virtues and their limitations. The new generation of ecological models are based on evolutionary branching, which provides a plausible mechanism for the generation of a stable regime of disruptive selection and the maintenance of polymorphism. These are important prerequisites for ecological differentiation and eventually the divergence into daughter species that can stably coexist since they inhabit different ecological niches. However, ecological differentiation is not sufficient for achieving speciation (Fig. 1). In view of the inherent complexity of speciation models, it is perhaps not surprising that 'branching models,' with their focus on the mechanisms underlying ecological differentiation, tended to give limited attention to the mechanisms underlying the evolution of a mating structure that leads to reproductive isolation. This is now changing gradually, and the mating process, in particular the costs of choosiness, receive more attention in the recent literature on ecological speciation (e.g., Matessi et al. 2001; Kirkpatrick and Nuismer 2004; Doebeli 2005; Schneider and Bürger 2006; Pennings et al. 2008; Kopp and Hermisson 2008; Ripa 2009). Yet, only Doebeli (2005) takes a mechanistic view on the process, and virtually all models consider the direct evolution of assortative mating. In comparison with direct assortative mating (which almost invariably induces some form of stabilising selection), diversifying selection on female preferences and associated male ornaments might be a more potent and more plausible 
Fig. 5 No speciation despite disruptive sexual selection. Without disruptive natural selection on bill size (green), polymorphisms in female mating preference (red) and male ornamentation (blue) are not maintained, due to competitive exclusion. In this case, we assumed a narrow ecological resource distribution that did not offer opportunities for ecological diversification. Adapted from Van Doorn and Weissing (2001)
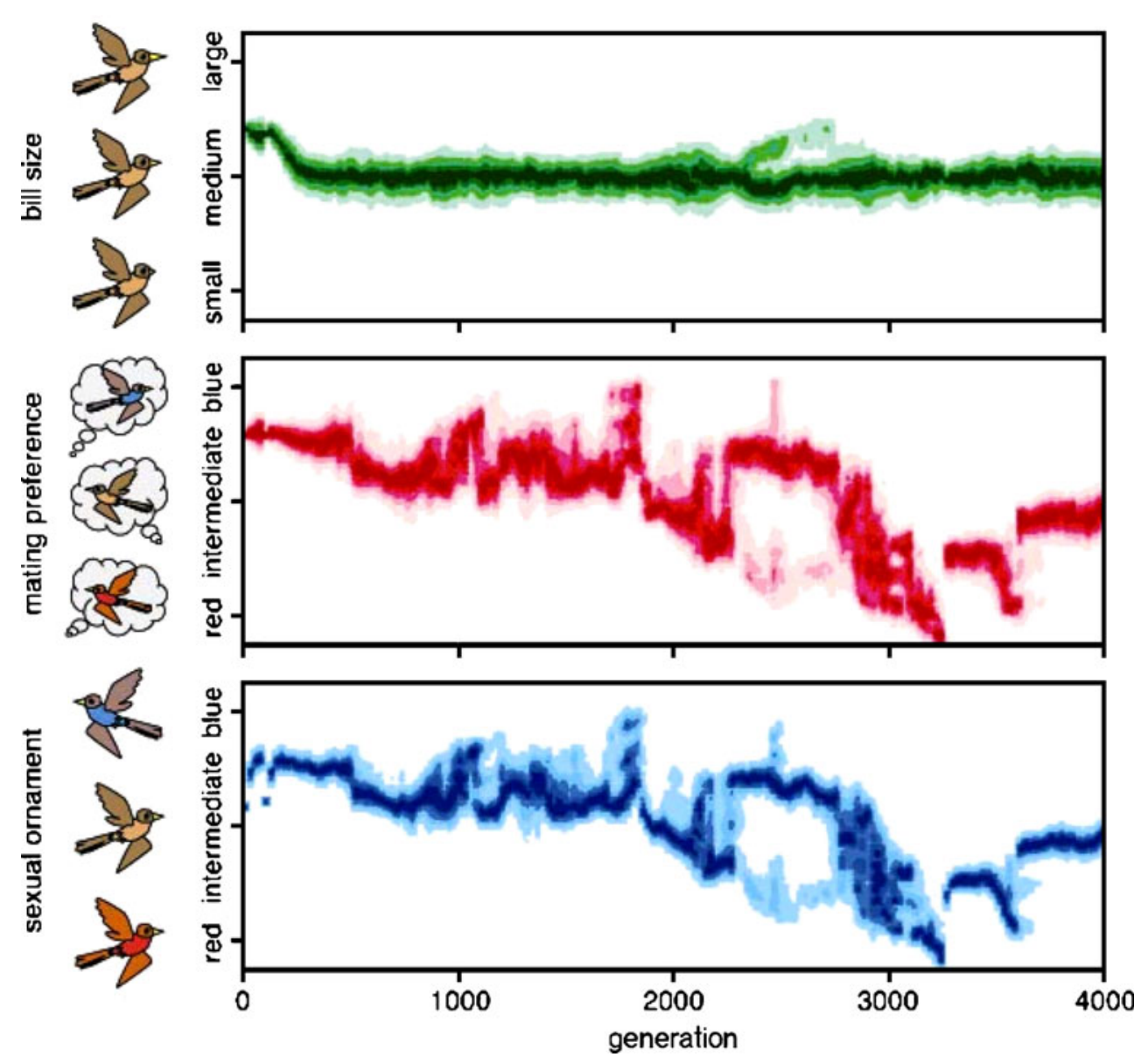

mechanism for achieving reproductive isolation. Sexual selection models of speciation have demonstrated that this may indeed be the case, provided that there are mechanisms leading to variation in female preferences. However, with a few exceptions, these models have neglected the necessity of ecological differentiation to achieve the stable coexistence of the incipient species.

Hence, ecological models of speciation require assortative mating, the evolution of which might be explained by sexual selection. On the other hand, sexual selection models require ecological differentiation for the coexistence of incipient species. Therefore, it has been argued (e.g., Galis and Metz 1998) that in real-world systems, speciation relies on the interplay of sexual selection and ecological processes. It is therefore surprising that rather few speciation models (e.g., Kondrashov and Kondrashov 1999; Van Doorn et al. 2001) include both disruptive natural and disruptive sexual selection. To our knowledge, the treatment of Van Doorn and Weissing (2001) is the only coherent study where the evolution of ecological differentiation through evolutionary branching and the divergence of mating preferences through Fisherian runaway selection is systematically investigated, both analytically and by means of computer simulations. Since this study is not easily accessible, we will discuss its main results in some detail.

In this model, an ecological character (in the figures represented by bill size) coevolves with a male ornament (e.g., plumage coloration) and female preferences for this trait. The ecological part of the model corresponds to that of the Dieckmann-Doebeli model (illustrated in Fig. 2) where evolutionary branching of the ecological character is to be expected whenever the resource distribution (e.g., the distribution of seed sizes) is broader than the resource utilisation curve (the spectrum of seeds that can be consumed by a bird with a given bill size). The sexual selection part of the model considers the evolution of a (costly) female preference for a (costly) male ornament. As illustrated in Fig. 3, evolutionary branching of the male ornament is to be expected if the distribution of female preferences is sufficiently broad. In the model, this can be achieved by a combination of weak selection and a large influx of new mutations: female preferences will become sufficiently variable if the costs of being choosy are small and if the distribution of new preference mutations is relatively broad. For reasons to be discussed below, the model considers the possibility that mating is not only determined by ornaments and preferences, but also by the 
degree of matching of the ecological character. The fixed level of assortative mating based on ecology turned out to be a model parameter of crucial importance.

Figure 4 shows a simulation where speciation occurs through the joint action of disruptive natural and sexual selection. In a first phase (the first 1,000 generations), the ecological character evolves toward its ecological optimum (here: medium bill size). Once this point is reached, the population experiences disruptive selection (the variation of bill sizes increases), but cannot undergo evolutionary branching, because assortative mating has not yet evolved. During this initial stage $(<1,500$ generations), male ornaments and female preferences evolve jointly toward that value of the ornament that maximises male survival. Several times (e.g., in generation 600), polymorphisms of ornaments and preferences originate, but these are unstable due to competitive exclusion and viability selection against extreme ornaments. After 1,500 generations, branching of ecotype and mating strategies repeatedly splits the population into groups that are ecologically differentiated and/or reproductively isolated. At first, these splitting events are only transient, until after about 3,000 generations a stable situation results, with two ecologically differentiated groups that are, at the same time, reproductively isolated. In the simulation shown, two daughter 'species' have evolved: a large-billed species with blue males and female preference for blue, and a small-billed species with red males and female preference for red.

It is not surprising that speciation only occurs if the conditions for ecotype branching (broad resource distribution, narrow resource utilisation curve) and for mating strategy branching (small costs of choosiness, sufficient influx of preference mutations) are both satisfied. Figure 5 illustrates what happens for parameter combinations that preclude the occurrence of ecotype branching. In comparison to Fig. 4, only the width of the resource utilisation curve was increased in relation to the resource distribution, implying that the consumer species is now an ecological generalist rather than a specialist. The upper panel in Fig. 5 shows that the species converges rapidly to its ecological optimum (a bill of medium size) and that it stays there with little variation because of stabilising selection. In contrast, the population is repeatedly split into reproductively isolated subpopulations with opposing mating strategies. However, these incipient daughter 'species' cannot stably coexist, because they are ecologically not differentiated but nevertheless have to compete for the same resources. In a situation like this, all but one of the subpopulations will be driven to extinction.

Figure 6 illustrates the situation when the conditions for ecotype branching are satisfied, but sexual selection does not become disruptive because the influx of new mutations is too low to sustain sufficient variation in female preferences. Disruptive selection on the ecological character leads to a broad distribution of bill sizes (Fig. 6, upper panel), but speciation does not occur, because stabilising selection on mating strategies precludes the evolution of assortative mating.

It is important to realise that the simultaneous occurrence of disruptive natural selection and disruptive sexual selection is necessary but not sufficient for selectiondriven speciation. Besides polymorphism of ecotypes and mating strategies, it is also required that, during speciation, linkage disequilibrium (i.e., genetic correlation) develops between ecological and mating strategies (Felsenstein 1981; Rice 1984). Only then will evolutionary branching of ecotype and mating type result in the evolution of reproductively isolated and - at the same time-ecologically differentiated species. This can be derived from Fig. 7, which depicts for the model considered here under which conditions speciation is to be expected. The figure was derived from the mathematical analysis of an adaptive dynamics approximation of the simulation model (for details, see Van Doorn and Weissing 2001). A quantitative genetics approximation yielded almost identical results.

From Fig. 7, it becomes apparent that speciation does not occur for parameter combinations where mating is solely dependent on sexual selection, that is, the match between female preferences and male ornaments (left-hand side of Fig. 7). In other words, for speciation to occur, disruptive natural and sexual selection have to be 'assisted' by at least some degree of assortative mating based on the ecological character. Otherwise, the required genetical correlation between ecological and mating strategies does apparently not evolve. However, in the presence of such assortative mating, the conditions for the evolution of ecological differentiation become more restrictive. This is apparent from the fact that the threshold for the strength of disruptive natural selection required to achieve evolutionary branching of the ecological character increases (linearly) with the degree of assortative mating. In other words, for branching to occur in the Dieckmann-Doebeli part of the model, the discrepancy between the width of the resource distribution and the width of the resource utilisation curve (see Fig. 2) has to be larger than the analysis in a randomly mating population would suggest. The same observation has been made in other models (e.g., Kirkpatrick and Nuismer 2004; Kopp and Hermisson 2008; Pennings et al. 2008; Ripa 2009), and it is explained by the fact that assortative mating induces stabilising selection when choosiness is costly. This stabilising selection counteracts and thus weakens disruptive selection on the ecological character and, hence, makes branching more difficult.

The analytical results underlying Fig. 7 were largely confirmed by extensive computer simulations (for details, see Van Doorn and Weissing 2001), in the sense that 
Fig. 6 No speciation despite disruptive natural selection. If females accept a broad range of mating partners, competition between males does not favour diversification among males. Rather, sexual selection favours the male ornament type (blue) that matches optimally with the most abundant female preference (red). Accordingly, there is no basis for reproductive isolation that could prevent interbreeding between individuals with different bill sizes (green). Although natural selection on bill size is disruptive, we, therefore, do not observe the emergence of two ecological specialists. Rather, disruptive selection leads to the maintenance of a broad unimodal distribution of bill sizes in the population. Adapted from Van Doorn and Weissing (2001)
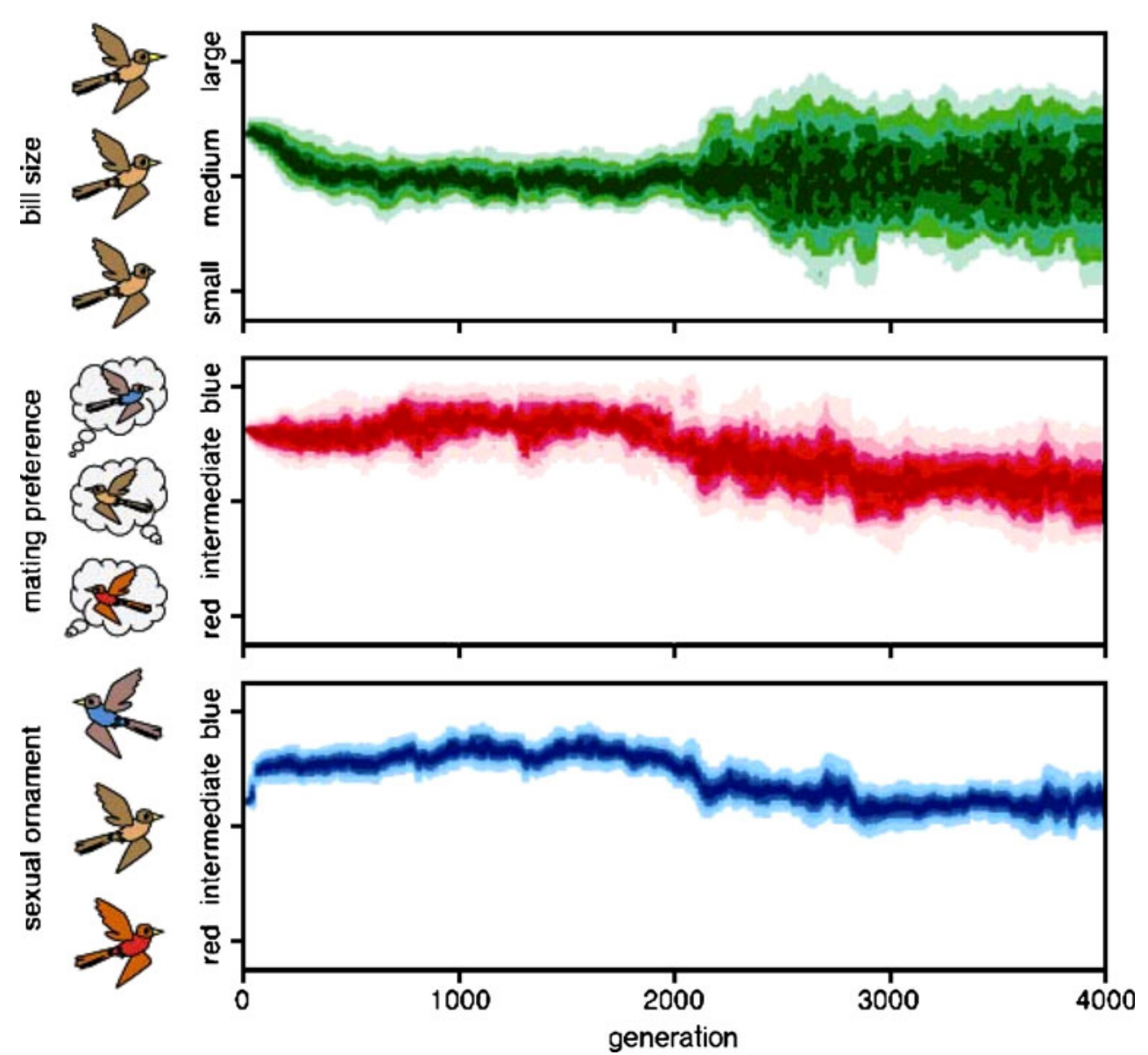

speciation did not occur when the simulation parameters were in the blue region of Fig. 7 ("no ecological diversification') and that the probability of speciation within a given time period was highest when the parameters were in the orange region ('speciation'). However, speciation regularly occurred in the yellow region ('maintenance of genetic variation in ecotype') as well and, in particular, also in the absence of assortative mating based on ecotype. This discrepancy can to a large part be explained by the stochasticity inherent in computer simulations, which allows the build-up of the genetic associations between ecological and mating strategies that are required for speciation (for details, see Van Doorn and Weissing 2001). By assuming an infinite population size, the analytical model neglects demographic and genetic stochasticity and therefore requires an external factor (here: some assortative mating based on ecotype) to accomplish a genetic association between ecological and mating strategies.

Ecological and sexual selection models are often viewed as contrasting approaches to speciation. In the empirical literature, repeatedly attempts are being made to determine whether speciation events are 'typically' driven by ecological factors or by sexual selection (e.g., Wilson et al. 2000; see also Panhuis et al. 2001; Schluter 2001; Ritchie 2007).
The model discussed above takes a more integrative perspective by viewing the evolution of ecological differentiation and the evolution of assortative mating are mutually dependent processes that are both required for adaptive speciation. Ecological differentiation arises naturally from evolutionary branching of competitive strategies (as determined by ecotype). Assortative mating results from evolutionary branching of male and female mating strategies. Speciation is completed when, in addition, a genetic association (i.e., linkage disequilibrium) develops between ecotypes and mating types, giving rise to reproductively isolated and, at the same time, ecologically differentiated daughter species.

\section{Are diverging preferences required for speciation?}

The results discussed above suggest that ecological specialisation and sexual selection have to work hand in hand in order to achieve adaptive speciation. From this perspective, it is somewhat paradoxical that virtually all sexual selection models of speciation are based on Fisherian runaway. Fisherian runaway selection is interesting from a theoretical point of view in that it demonstrates how 


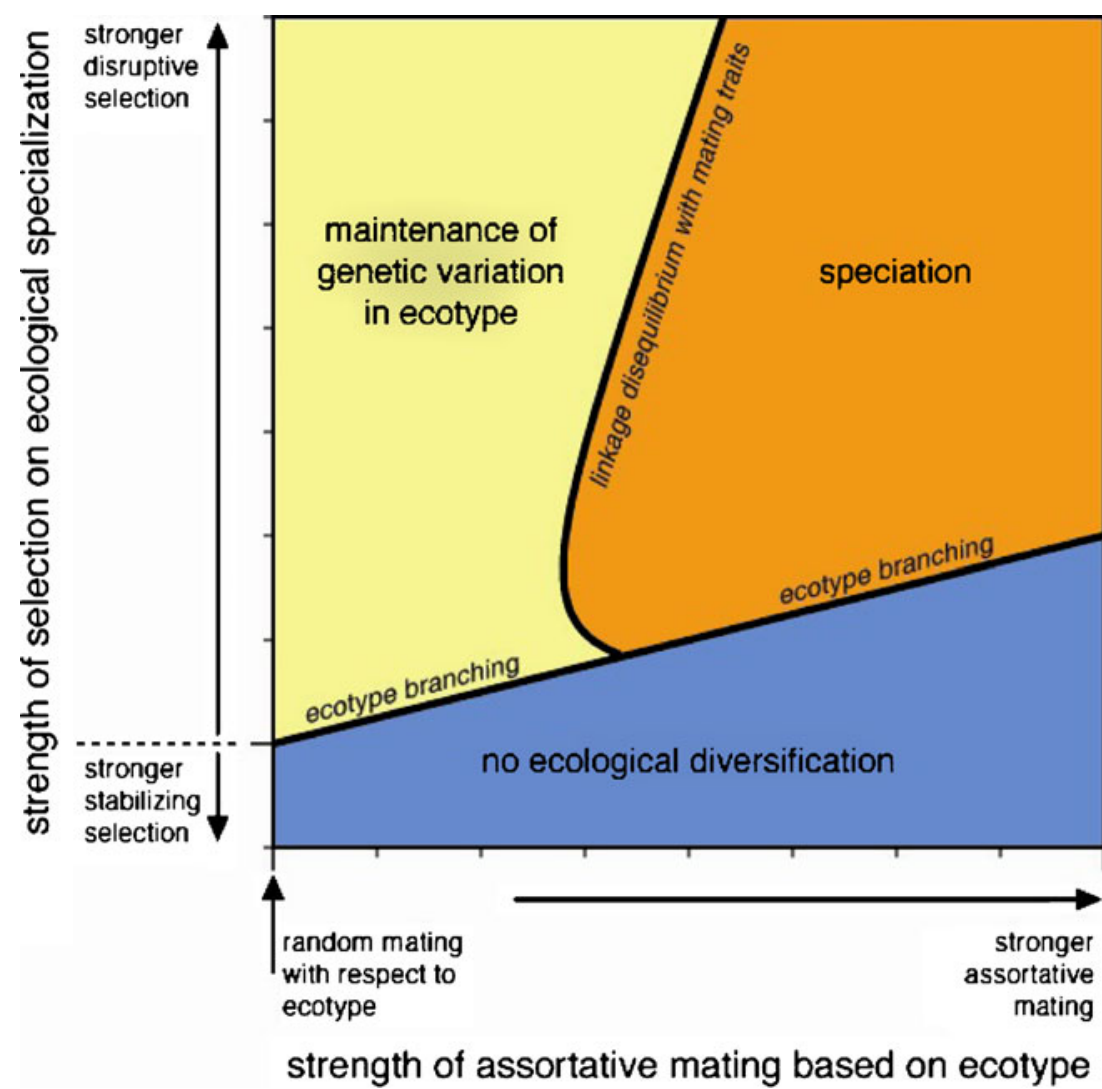

Fig. 7 Parameter regime leading to speciation through the interaction of natural and sexual selection. Disruptive selection on ecological characters (bill size) and mating traits (female preference and male ornament), is a necessary but not sufficient condition for speciation in the model by Van Doorn and Weissing (2001). The evolution of reproductively isolated and stably coexisting species requires that the genes responsible for reproductive isolation become associated with the genes responsible for ecological divergence (this occurs in the orange area). Such an association does not easily arise by chance, since recombination tends to rapidly break down any non-random association between genes.

preferences can evolve for ornaments that are quite arbitrary and in fact a burden for their carriers as far as natural (i.e., viability) selection is concerned. In contrast to Fisherian runaway, where natural and sexual selection may be viewed as opposing forces, the two flavors of selection work much more in concert when other forms of sexual selection are considered. This is in particular the case when female preferences are targeted at male characters that signal a high genetic quality (good genes sexual selection) or the ability to supply resources like paternal care (direct benefits sexual selection). When male ornaments are indicators of high genetic or phenotypic quality, then sexual selection is automatically interlocked with natural selection, and one might therefore expect that good genes or direct benefit sexual selection could boost disruptive natural selection in driving speciation.

There is, however, a good reason why sexual selection models have focused on Fisherian runaway selection.
However, if mating is partially based on ecological characters (for example, individuals with similar bill sizes may forage in the same areas, and may therefore be more likely to mate), then this can provide a counterforce against the erosion of associations between genes by recombination. A minimal strength of the effect of ecological differences on mating (the horizontal axis reflects the specificity of selfreferent phenotype matching based on bill size), is necessary for speciation to occur (orange area) in combination with sufficiently strong disruptive selection (vertical axis)

Virtually all these models sought a role of sexual selection as a driving force behind the divergence of mating strategies. Such a divergence is not implausible in case of Fisherian runaway selection: since a Fisherian runaway process can be based on any arbitrary male trait, it easy to imagine that it runs in different directions in different (daughter) populations. The situation is fundamentally different in case of good genes or direct benefit sexual selection. Here the male ornament under focus has a 'meaning,' since it acts as an indicator of genetic or phenotypic quality (Andersson 1994). Accordingly, mate choice based on such indicator traits has a clear directionality, making it difficult to conceive how sexual selection could lead to the divergence of preferences between two nascent species.

Recently, we demonstrated by means of a good genes model that the divergence of mating preferences is actually not required to give sexual selection a crucial role in 
adaptive speciation (Van Doorn et al. 2009). The idea behind this model is illustrated in Fig. 8. Imagine a patchy environment where the habitat patches differ in ecological conditions (here symbolised by seeds of different size). The individuals of a consumer species may differ in their food exploitation strategy (here indicated by bill size), analogously to the situation in Fig. 2. In the situation depicted in Fig. 8, a small-billed bird has the highest food intake (and hence the highest viability) in habitat 1 , while a large-billed bird has the highest food intake in habitat 2. We assume that birds with medium bill size perform worse when averaged over both habitats than either small- or largebilled birds. Hence natural selection is stabilising within habitats and disruptive at the level of the entire population. Computer simulations (based on Levene's soft selection model; Levene 1953) show that in the presence of gene flow between the two habitats disruptive selection does not easily result in speciation. Although selection constantly removes individuals with intermediate phenotypes (medium bill sizes) from the population, such individuals are created anew every generation as a result of migration between the habitats and recombination between different specialist genotypes. Even if migration between the habitats is limited, gene flow between habitats nevertheless prevents the population from splitting into two locally adapted species, unless disruptive selection is unusually strong.

The situation changes if we consider the possibility of sexual selection for an ornament (red plumage color in Fig. 8) that is expressed in a condition-dependent manner. In natural systems, this is a common situation (e.g., Griffith et al. 1999; Moczek and Emlen 1999; David et al. 2000; Hill 2000; Jensen et al. 2006; Miller and Emlen 2010). In the scenario considered here, individuals differ in their degree of local adaptation. If this is the case, a conditiondependent ornament functions as an indicator for the degree of local adaptation, since only those individuals that welladapted to the local environment will be in a good condition and, hence, able to develop a bright plumage (in Fig. 8, the short-billed birds in habitat 1 and the longbilled birds in habitat 2). Once a female preference for such an indicator of local adaptation has arisen, it can readily spread in the population. In fact, females benefit from mating with locally adapted males, because on average, this decreases the probability of producing offspring with intermediate phenotype, which in this model have a lower fitness. A preference for locally adapted mates is even more advantageous when offspring are more likely to end up in the same habitat as the parents (for example, when individuals are philopatric to some degree) or exert matching habitat choice on the basis of their ecological phenotype (Edelaar et al. 2008). It is therefore not surprising that-in line with earlier models (Proulx 2001; Lorch et al. 2003; Reinhold 2004) — mate choice based on a condition-dependent ornament does readily evolve.

This has important implications for speciation. First, sexual selection intensifies disruptive natural selection. Males which are ecologically not well adapted to the local conditions (e.g., a large-billed bird in habitat 1) do not only have an ecological disadvantage, but also a mating disadvantage, since they are not are not in the condition to develop the bright plumage preferred by females. Second, sexual selection creates or strengthens assortative mating with respect to the ecological strategy, since it reduces the rate of interbreeding between specialists for

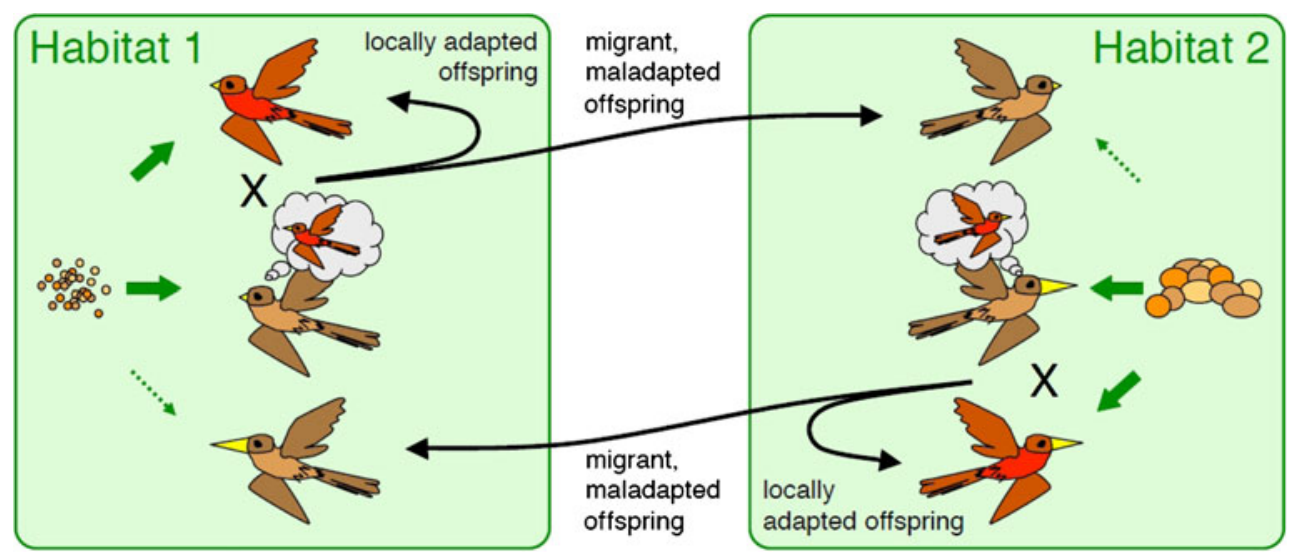

Fig. 8 Speciation through the joint action of disruptive natural selection and sexual selection for an ornament signalling local adaptation. Two habitats differing in ecological conditions (symbolized by seed size) exert natural selection on an ecological character (bill size). Selection is disruptive, since birds with intermediate bill size have averaged over habitats, a lower viability that the birds specialised on one of the two habitats. Yet, speciation will only occur if migration between habitats is very low and/or selection against intermediate types is very intense. This changes when selection for a condition-dependent ornament (red plumage) is added to the model. Since only birds in good condition are able to express the ornament (small-billed birds in habitat 1, large-billed birds in habitat 2), the ornament acts as an indicator of local adaptation. Although female preferences do not differ in the two habitats, sexual selection intensifies disruptive natural selection and facilitates the evolution of reproductive isolation 
different habitats. In the rare event that habitat specialists do interbreed, sexual selection effectively removes 'hybrid' sons from the mating pool, since these sons are in poor condition in either habitat and therefore not able to develop the bright plumage required to attract mates.

Thus, mate choice based on a condition-dependent ornament intensifies both disruptive natural selection and assortative mating. On the other hand, disruptive natural selection favours the evolution of (costly) female preferences, since it creates and maintains variation in genetic quality. Without such variation, the benefits or being choosy are small and easily dominated by the costs of choice (the 'lek paradox;' Kotiaho et al. 2001; Kokko and Heubel 2008). Accordingly, natural and sexual selection work hand in hand, mutually reinforcing each other and paving the way to adaptive speciation (Fig. 9). While pure ecological speciation only occurs under rather extreme conditions (strong disruptive selection, strong tendency towards philopatry), the conditions allowing for selectiondriven speciation are considerably relaxed once sexual selection is added to the model (for details, see Van Doorn et al. 2009; for a similar model based on local adaptation of the immune system see Eizaguirre et al. 2009).

We can conclude that the divergence of preferences and mating signals is not really required to give sexual selection

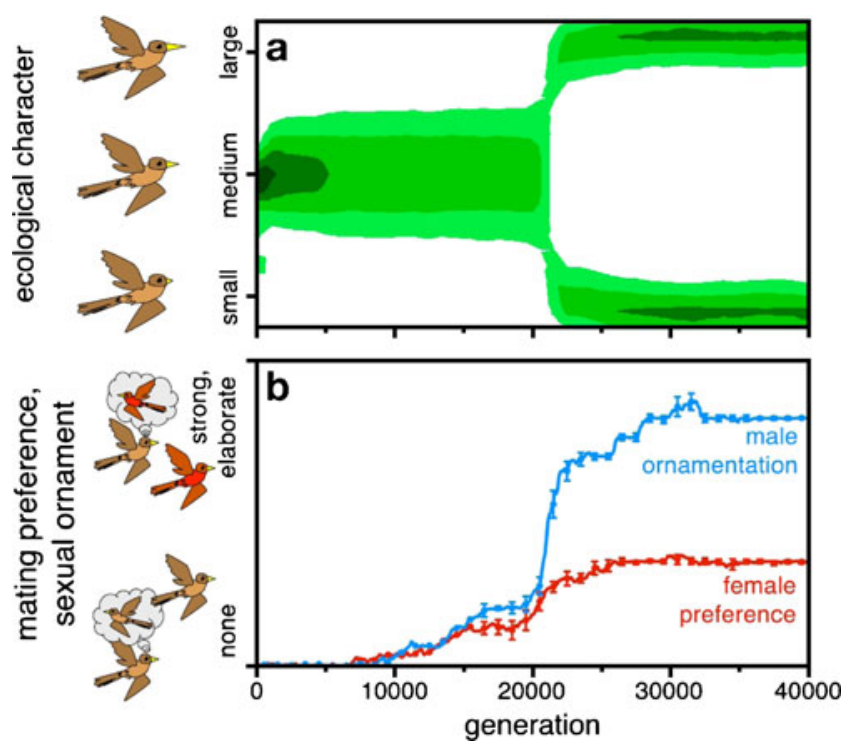

Fig. 9 Sexual selection facilitates speciation through disruptive natural selection even in the absence of diverging preferences (after Van Doorn et al. 2009). In the absence of sexual selection, disruptive selection for ecological specialisation will typically not lead to speciation, but to a broad distribution of ecological characters (bill sizes). The variation in ecological characters, many of which not well adapted to local circumstances, creates favourable conditions for the spread of costly female preferences for a costly condition-dependent male ornament (plumage colour). Once this preference has evolved, natural and sexual selection mutually reinforce each other, eventually leading to reproductively isolated ecological specialist species an important role in adaptive speciation. For several reasons, good-genes (or direct-benefit) sexual selection based on condition-dependent ornaments seems to be better suited to resolve the core problems of adaptive speciation theory than disruptive sexual selection based on diverging runaway processes. First, local adaptation and condition-dependent mating signals are ubiquitous in natural populations (e.g., Griffith et al. 1999; Moczek and Emlen 1999; Qvarnström 1999; David et al. 2000; Hill 2000; Dolgin et al. 2006; Jensen et al. 2006; Miller and Emlen 2010; but see Cotton et al. 2004). The idea that sexual ornaments signal the degree of local adaptation is therefore plausible and applicable to many natural systems (e.g., Hunt et al. 2004; Kokko and Heubel 2008). Second, when the environment is heterogeneous, sexual selection for condition-dependent ornaments can easily get off the ground (Proulx 2001; Lorch et al. 2003; Reinhold 2004; Van Doorn et al. 2009). In contrast, diverging runaway selection requires a broad variation in female preferences, which - as discussed in detail above - is not easy to achieve. Third, the interaction of disruptive ecological selection and sexual selection for local adaptation does not suffer from one of the main obstacles to speciation: the evolution of sufficiently strong genetic associations between ecological and mating strategies (Arnegard and Kondrashov 2004; Gavrilets 2004; Bolnick and Fitzpatrick 2007). Since the same type of preference spreads in both nascent species, the evolution of assortative mating and reproductive isolation depends on a "one-allele" mechanism sensu Felsenstein (1981). It is well-acknowledged in the speciation literature that speciation is much more easy to achieve in "one-allele" models than in "two-allele" models.

The model of Van Doorn et al. (2009) does not directly explain why closely related species tend to differ most markedly with respect to mating traits and secondary sexual characters (e.g. Panhuis et al. 2001; Ritchie 2007). In contrast to sexual selection models based on diverging mating strategies, the same kind of preference (in Fig. 8: a preference for a red plumage) is present in both nascent species. In other words, the resulting daughter species are more cryptic than in traditional models of speciation by sexual selection. However, this may only be a transient state. Once the incipient daughter species become reproductively isolated, their evolution is also becoming decoupled, allowing the accumulation of differences in mating characters, for instance via arms races between the sexes caused by sexual conflict (Parker and Partridge 1998; Gavrilets 2000) or by (separate) runaway processes in one or several populations.

\section{Concluding remarks}

Due to theoretical developments in the past two decades, adaptive speciation (that is, speciation fully driven by 
natural and/or sexual selection) has become much more plausible than traditional models seem to suggest. The concept of evolutionary branching reveals how a stable regime of disruptive natural selection can be created and maintained in a plausible and realistic way. However, ecological models of speciation still suffer from the problem that assortative mating leading to reproductive isolation will only evolve under rather specific conditions. Sexual selection models can, in principle, explain the divergence of mating strategies and, hence, the evolution of reproductive isolation. However, most of these models rely on the simultaneous occurrence of two Fisherian runaway processes, which will only occur in the presence of large variation in female preferences. Although various processes can produce the required variation (e.g., mutation-selection balance; disruptive selection on both female preferences and male traits), their prerequisites seem too restricted to view disruptive sexual selection as a general mechanism driving the evolution of reproductive isolation under a broad range of conditions. Even if disruptive sexual selection leads to reproductive isolation, additional factors are required to guarantee the stable coexistence of the nascent species. In principle, ecological differentiation allowing stable coexistence and reproductive isolation preventing interbreeding can both be achieved if disruptive natural selection and disruptive sexual selection are acting at the same time. Adaptive speciation in the purest sense of the word is indeed feasible under this scenario, but again under highly restrictive conditions. In particular, it is not sufficient that the conditions for the divergence of ecological and mating strategies are both satisfied. In addition, there has to be a mechanism allowing the evolution of a genetic association of the genes underlying ecological and mating strategies.

In view of the above constraints, many evolutionary biologists are still sceptical towards the idea of adaptive speciation. By means of a model not requiring diverging mating strategies we have shown, however, that this scepticism may not be justified. Speciation driven by the joint action of natural and sexual selection may indeed be unlikely if both processes are completely decoupled, as most present-day models tend to assume. Many obstacles on the road to speciation can be overcome, however, if one realises that not only natural selection but also sexual selection is strongly dependent on environmental conditions (Endler 1992). As a consequence, natural and sexual selection can favour the same traits, thereby mutually reinforcing each other (as in the case of conditiondependent ornaments discussed above). Even if this is not the case, it is conceivable that environmental differences create the genetic associations between ecological and mating strategies required for speciation if different combinations of ecotypes and mating types are favoured in different environments (e.g., Kawata et al. 2007). This requires environment-dependent natural selection on the one hand and environment-dependent sexual selection on the other. In our opinion, the final word on the empirical relevance of adaptive speciation theory has not yet been spoken.

Acknowledgements We thank Thor Veen for many stimulating discussions and Roger Butlin and the referees for their useful comments. FJW would like to thank the members of the ethological research station Hasli (University of Bern) for a pleasant and productive stay, during which part of this paper was written.

Conflicts of interest The authors declare that they have no conflict of interest.

Open Access This article is distributed under the terms of the Creative Commons Attribution Noncommercial License which permits any noncommercial use, distribution, and reproduction in any medium, provided the original author(s) and source are credited.

\section{References}

Abrams PA, Harada Y, Matsuda H (1993a) On the relationship between quantitative genetic and ESS models. Evolution 47:982-985

Abrams PA, Matsuda H, Harada Y (1993b) Evolutionarily unstable fitness maxima and stable fitness minima of continuous traits. Evol Ecol 7:465-487

Almeida CR, de Abreu FV (2003) Dynamical instabilities lead to sympatric speciation. Evol Ecol Res 5:739-757

Andersson M (1994) Sexual selection. Princeton University Press, Princeton

Arnegard ME, Kondrashov AS (2004) Sympatric speciation by sexual selection alone is unlikely. Evolution 58:222-237

Baker JM (2005) Adaptive speciation: the role of natural selection mechanisms of geographic and non-geographic speciation. Stud Hist Philos Biol Biomed Sci 36:303-326

Berner D, Adams DC, Grandchamp AC, Hendry AP (2008) Natural selection drives patterns of lake-stream divergence in stickleback foraging morphology. J Evol Biol 21:1653-1665

Bolnick DI (2004) Waiting for sympatric speciation. Evolution 58:895-899

Bolnick DI, Doebeli M (2003) Sexual dimorphism and adaptive speciation: two sides of the same ecological coin. Evolution 57:2433-2449

Bolnick DI, Fitzpatrick BM (2007) Sympatric speciation: models and empirical evidence. Ann Rev Ecol Evol Syst 38:459-487

Bürger R, Schneider KA, Willensdorfer M (2006) The conditions for speciation through intraspecific competition. Evolution 60:2185-2206

Cotton S, Fowler K, Pomiankowski A (2004) Do sexual ornaments demonstrate heightened condition-dependent expression as predicted by the handicap hypothesis? Proc Roy Soc Lond B 271:771-783

Coyne JA, Orr HA (2004) Speciation. Sinauer Associates, Sunderland

Darwin C (1859) On the origin of species by means of natural selection, or the preservation of favoured races in the struggle for life. John Murray, London

Darwin C (1871) The descent of man, and selection in relation to sex. John Murray, London

David P, Bjorksten T, Fowler K, Pomiankowski A (2000) Conditiondependent signalling of genetic variation in stalk-eyed flies. Nature 406:186-188 
Day $\mathrm{T}$ (2005) Modelling the ecological context of evolutionary change: déjà vu or something new? In: Cuddington $\mathrm{K}$, Beisner $\mathrm{BE}$ (eds) Ecological paradigms lost: routes to theory change. Academic, New York

Dieckmann U, Doebeli M (1999) On the origin of species by sympatric speciation. Nature 400:354-357

Dieckmann U, Doebeli M, Metz JAJ, Tautz D (eds) (2004) Adaptive speciation. Cambridge University Press, Cambridge

Dobzhansky T (1940) Speciation as a stage in evolutionary divergence. Am Nat 74:312-321

Doebeli M (2005) Adaptive speciation when assortative mating is based on female preference for male marker traits. J Evol Biol 18:1587-1600

Doebeli M, Dieckmann U (2000) Evolutionary branching and sympatric speciation caused by different types of ecological interactions. Am Nat 156:S77-S101

Doebeli M, Dieckmann U (2005) Adaptive dynamics as a mathematical tool for studying the ecology of speciation processes. J Evol Biol 18:1194-1200

Doebeli M, Dieckmann U, Metz JAJ, Tautz D (2005) What we have also learned: adaptive speciation is theoretically plausible. Evolution 59:691-695

Dolgin ES, Whitlock MC, Agrawal AF (2006) Male Drosophila melanogaster have higher mating success when adapted to their thermal environment. J Evol Biol 19:1894-1900

Edelaar P, Siepielski AM, Clobert J (2008) Matching habitat choice causes directed gene flow: a neglected dimension in evolution and ecology. Evolution 62:2462-2472

Eizaguirre C, Lenz TL, Traulsen A, Milinski M (2009) Speciation accelerated and stabilized by pleiotropic major histocompatibility immunogenes. Ecol Lett 12:5-12

Endler JA (1992) Signals, signal conditions, and the direction of evolution. Am Nat 139:S125-S153

Felsenstein J (1981) Skepticism towards Santa Rosalia, or why are there so few kinds of animals? Evolution 35:124-138

Fisher RA (1930) The genetical theory of natural selection. Clarendon, Oxford, pp 135-162 (2nd ed., 1958, Dover, New York)

Galis F, Metz JAJ (1998) Why are there so many cichlid species? Trends Ecol Evol 13:1-2

Gavrilets S (1999) A dynamical theory of speciation on holey adaptive landscapes. Am Nat 154:1-22

Gavrilets S (2000) Rapid evolution of reproductive barriers driven by sexual conflict. Nature 403:886-889

Gavrilets S (2003) Models of speciation: what have we learnt in 40 years? Evolution 57:2197-2215

Gavrilets S (2004) Fitness landscapes and the origin of species. Princeton University Press, Princeton

Gavrilets S (2005) "Adaptive speciation"-it is not that easy: a reply to Doebeli et al. Evolution 59:696-699

Gavrilets S, Vose A (2007) Case studies and mathematical models of ecological speciation. 2. Palms on an oceanic island. Mol Ecol 16:2910-2921

Gavrilets S, Vose A, Barluenga M, Salzburger W, Meyer A (2007) Case studies and mathematical models of ecological speciation. 1. Cichlids in a crater lake. Mol Ecol 16:2893-2909

Geritz SAH, Kisdi E, Meszéna G, Metz JAJ (1998) Evolutionarily singular strategies and the adaptive growth and branching of the evolutionary tree. Evol Ecol Res 12:35-57

Geritz SAH, Kisdi E, Meszéna G, Metz JAJ (2004) Adaptive dynamics of speciation: ecological underpinnings. In: Dieckmann U, Doebeli M, Metz JAJ, Tautz D (eds) Adaptive speciation. Cambridge University Press, Cambridge, pp 54-75

Gourbiere S (2004) How do natural and sexual selection contribute to sympatric speciation? J Evol Biol 17:1297-1309

Griffith SC, Owens IPF, Burke T (1999) Environmental determination of a sexually selected trait. Nature 400:358-360
Higashi M, Takimoto G, Yamamura N (1999) Sympatric speciation by sexual selection. Nature 402:523-526

Hill GE (2000) Energetic constraints on expression of carotenoidbased plumage coloration. J Avian Biol 31:559-566

Hunt J, Bussière LF, Jennions MD, Brooks R (2004) What is genetic quality? Trends Ecol Evol 19:329--333

Jensen H, Svorkmo-Lundberg T, Ringsby TH, Sæther BE (2006) Environmental influence and cohort effects in a sexual ornament in the house sparrow, Passer domesticus. Oikos 114:212-224

Johansson J, Ripa J (2006) Will sympatric speciation fail due to stochastic competitive exclusion? Am Nat 168:572-578

Kawata M, Shoji A, Kawamura S, Seehausen O (2007) A genetically explicit model of speciation by sensory drive within a continuous population in aquatic environments. BMC Evol Biol 7:99

Kawecki T (2004) Genetic theories of sympatric speciation. In: Dieckmann U, Doebeli M, Metz JAJ, Tautz D (eds) Adaptive speciation. Cambridge University Press, Cambridge, pp 36-53

Kirkpatrick M, Nuismer SL (2004) Sexual selection can constrain sympatric speciation. Proc Roy Soc Lond B 271:687-693

Kirkpatrick M, Ravigné V (2002) Speciation by natural and sexual selection: models and experiments. Am Nat 158:S22-S35

Kirkpatrick M, Servedio MR (1999) The reinforcement of mating preferences on an island. Genetics 151:865-884

Kokko H, Heubel K (2008) Condition-dependence, genotype-byenvironment interactions and the lek paradox. Genetica 132:209216

Kondrashov AS, Kondrashov FA (1999) Interactions among quantitative traits in the course of sympatric speciation. Nature 400:351-354

Kopp M, Hermisson J (2008) Competitive speciation and costs of choosiness. J Evol Biol 21:1005-1023

Kotiaho JS, Simmons LW, Tomkins JL (2001) Towards a resolution of the lek paradox. Nature 410:684-686

Labonne J, Hendry AP (2010) Natural and sexual selection giveth and taketh away reproductive barriers: models of population divergence in guppies. Am Nat 176:26-39

Lande R (1981) Models of speciation by sexual selection. Proc Nat Acad Sci USA 78:3721-3725

Lande R (1982) Rapid origin of sexual isolation and character divergence in a cline. Evolution 36:213-223

Lande R, Kirkpatrick M (1988) Ecological speciation by sexual selection. J Theor Biol 133:85-98

Lande R, Seehausen O, van Alphen JJM (2001) Mechanisms of rapid sympatric speciation by sex reversal and sexual selection in cichlid fish. Genetica 112-113:435-443

Levene H (1953) Genetic equilibrium when more than one ecological niche is available. Am Nat 87:331-333

Levins R (1966) Strategy of model building in population biology. Amer Sci 54:421-431

Lorch PD, Proulx S, Rowe L, Day T (2003) Condition-dependent sexual selection can accelerate adaptation. Evol Ecol Res 5:867-881

Marshall J, McNamara J, Houston A (2010) The state of Darwinian theory. Behav Ecol Sociobiol. doi:10.1007/s00265-010-1121-y

Matessi C, Gimelfarb A, Gavrilets S (2001) Long-term buildup of reproductive isolation promoted by disruptive selection: how far does it go? Selection 2:41-64

Maynard Smith J (1966) Sympatric speciation. Am Nat 100:637-650

Mayr E (1942) Systematics and the origin of species. Harvard University Press, Cambridge

Mayr E (1963) Animal species and evolution. Belknap, Cambridge

Mayr E, Provine WB (eds) (1998) The evolutionary synthesis: perspectives on the unification of biology. Harvard University Press, Cambridge

McNamara JM, Weissing FJ (2010) Evolutionary game theory. In: Székeley T, Moore AJ, Komdeur J (eds) Social behaviour. Genes, ecology and evolution. Cambridge University Press, Cambridge, pp 109-133 
Metz JAJ (2008) Fitness. In: Jorgensen SE, Fath BD (eds) Encyclopedia of ecology. Elsevier, Amsterdam

Miller CW, Emlen DJ (2010) Dynamic effects of oviposition site on offspring sexually-selected traits and scaling relationships. Evol Ecol 24:375-390

Moczek AP, Emlen DJ (1999) Proximate determination of male horn dimorphism in the beetle Onthophagus taurus (Coleoptera: Scarabaeidae). J Evol Biol 12:27-37

Nosil P, Yukilevich R (2008) Mechanims of reinforcement in natural and simulated polymorphic populations. Biol J Linn Soc 95:305-319

Orr HA (1995) The population genetics of speciation: the evolution of hybrid incompatibilities. Genetics 139:1805-1813

Orr HA, Turelli M (2001) The evolution of postzygotic isolation: accumulating Dobzhansky-Muller incompatibilities. Evolution 55:1085-1094

Panhuis TM, Butlin R, Zuk M, Tregenza T (2001) Sexual selection and speciation. TREE 16:364-371

Parker GA, Partridge L (1998) Sexual conflict and speciation. Philos Trans R Soc Lond B 353:261-274

Pennings PS, Kopp M, Meszéna G, Dieckmann U, Hermisson J (2008) An analytically tractable model for competitive speciation. Am Nat 171:E44-E71

Polechová J, Barton NH (2005) Speciation through competition: a critical review. Evolution 59:1194-1210

Presgraves DC (2010) The molecular evolutionary basis of species formation. Nat Rev Genet 11:175-180

Proulx SR (2001) Female choice via indicator traits easily evolves in the face of recombination and migration. Evolution 55:2401-2411

Queiroz D (2005) Ernst Mayr and the modern concept of species. Proc Natl Acad Sci USA 102:6600-6607

Qvarnström A (1999) Genotype-by-environment interactions in the determination of the size of a secondary sexual character in the collared flycatcher (Ficedula albicollis). Evolution 53:1564-1572

Reinhold K (2004) Modeling a version of the good-genes hypothesis: female choice of locally adapted males. Org Divers Evol 4:157163

Rice WC (1984) Disruptive selection on habitat preferences and the evolution of reproductive isolation: a simulation study. Evolution 38:1251-1260

Ripa J (2009) When is sympatric speciation truly adaptive? An analysis of the joint evolution of resource utilization and assortative mating. Evol Ecol 23:31-52

Ritchie MG (2007) Sexual selection and speciation. Ann Rev Ecol Evol Syst 38:79-102

Rosenzweig ML (1978) Competitive speciation. Biol J Linn Soc 10:275-289

Rueffler C, van Dooren TJM, Leimar O, Abrams PA (2006) Disruptive selection and then what? Trends Ecol Evol 21:238-245

Sadedin S, Hollander J, Panova M, Johannesson K, Gavrilets S (2009) Case studies and mathematical models of ecological speciation. 3: Ecotype formation in a Swedish snail. Mol Ecol 18:4006-4023
Schluter D (2001) Ecology and the origin of species. Trends Ecol Evol 16:372-380

Schneider KA, Bürger R (2006) Does competitive divergence occur if assortative mating is costly? J Evol Biol 19:570-588

Seehausen O, van Alphen JJM, Witte F (1997) Cichlid fish diversity threatened by eutrophication that curbs sexual selection. Science 277:1808-1811

Servedio MR (2004) The what and why of research on reinforcement. PLoS Biol 2:2032-2035

Servedio MR, Noor MAF (2003) The role of reinforcement in speciation: theory and data. Ann Rev Ecol Evol Syst 34:339-364

Takimoto G (2002) Polygenic inheritance is not necessary for sympatric speciation by sexual selection. Popul Ecol 44:87-91

Takimoto G, Higashi M, Yamamura N (2000) A deterministic genetic model for sympatric speciaton by sexual selection. Evolution $54: 1870-1881$

Turelli M, Barton NH, Coyne JA (2001) Theory and speciation. TREE 16:330-343

Turner GF, Burrows MT (1995) A model of sympatric speciation by sexual selection. Proc Roy Soc Lond B 260:287-292

Udovic D (1980) Frequency-dependent selection, disruptive selection, and the evolution of reproductive isolation. Am Nat 116:621-641

Unckless RL, Orr HA (2009) Dobzhansky-Muller incompatibilities and adaptation to a shared environment. Heredity 102:214-217

Van Dooren TJM, Demon I, Durinx M (2004) Sexual dimorphism or evolutionary branching? Evol Ecol Res 6:857-871

Van Doorn GS (2004) Sexual Selection and Sympatric Speciation. Ph.D. thesis, University of Groningen. Available at http://dissertations.ub. rug.nl/faculties/science/2004/g.s.van.doorn/

Van Doorn GS, Weissing FJ (2001) Ecological versus sexual selection models of sympatric speciation: a synthesis. Selection 2:17-40

Van Doorn GS, Noest AJ, Hogeweg P (1998) Sympatric speciation and extinction driven by environment dependent sexual selection. Proc R Soc Lond B 265:1915-1919

Van Doorn GS, Luttikhuizen PC, Weissing FJ (2001) Sexual selection at the protein level drives the extraordinary divergence of sex related genes during sympatric speciation. Proc R Soc Lond B 268:2155-2161

Van Doorn GS, Dieckmann U, Weissing FJ (2004) Sympatric speciation by sexual selection: a critical re-evaluation. Am Nat 163:709-725

Van Doorn GS, Edelaar P, Weissing FJ (2009) On the origin of species by natural and sexual selection. Science 326:1704-1707

Waxman D, Gavrilets S (2005) 20 questions on adaptive dynamics. J Evol Biol 18:1139-1154

Wilson AB, Noack-Kunnmann K, Meyer A (2000) Incipient speciation in sympatric Nicaraguan crater lake cichlid fishes: sexual selection versus ecological diversification. Proc Roy Soc Lond B 267:2133-2141

Wu CI (1985) A stochastic simulation study on speciation by sexual selection. Evolution 39:66-82 